\title{
Adsorption and Conformation of Serum Albumin Protein on Gold Nanoparticles Investigated Using Dimensional Measurements and in Situ Spectroscopic Methods
}

\author{
De-Hao Tsai, ${ }^{\dagger}$ Frank W. DelRio, $^{\dagger}$ Athena M. Keene, ${ }^{\ddagger}$ Katherine M. Tyner, $^{\dagger}$ Robert I. MacCuspie, $^{\dagger}$ \\ Tae Joon Cho, ${ }^{\dagger}$ Michael R. Zachariah, ${ }^{+, \S}$ and Vincent A. Hackley ${ }^{*+}$ \\ ${ }^{\dagger}$ Material Measurement Laboratory, National Institute of Standards and Technology, Gaithersburg, Maryland 20899-8520, \\ United States \\ ${ }^{\ddagger}$ Center for Drug Evaluation and Research, Food and Drug Administration, Silver Spring, Maryland 20993, United States \\ ${ }^{\S}$ Departments of Mechanical Engineering and Chemistry, University of Maryland, College Park, Maryland 20740, United States \\ Supporting Information
}

\begin{abstract}
The adsorption and conformation of bovine serum albumin (BSA) on gold nanoparticles (AuNPs) were interrogated both qualitatively and quantitatively via complementary physicochemical characterization methods. Dynamic light scattering (DLS), asymmetric-flow field flow fractionation (AFFF), fluorescence spectrometry, and attenuated total reflectanceFourier transform infrared (ATR-FTIR) spectroscopy were combined to characterize BSA-AuNP conjugates under fluid conditions, while conjugates in the aerosol state were characterized by electrospray-differential mobility analysis (ES-DMA).

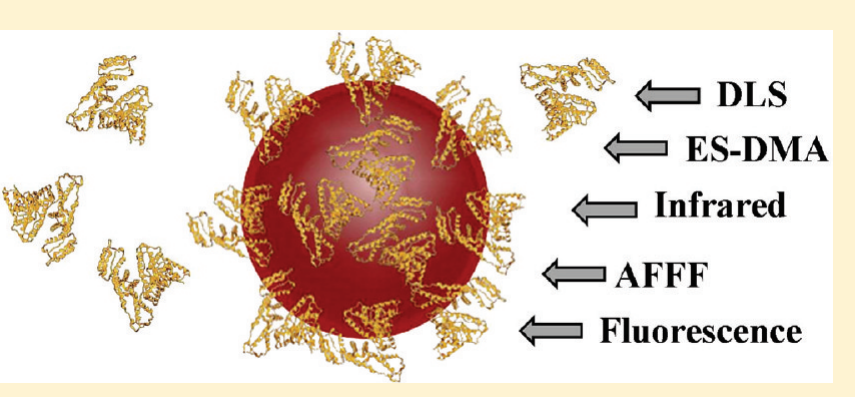
The presence of unbound BSA molecules interferes with DLS analysis of the conjugates, particularly as the AuNP size decreases (i.e., below $30 \mathrm{~nm}$ in diameter). Under conditions where the $\gamma$ value is high, where $\gamma$ is defined as the ratio of scattering intensity by AuNPs to the scattering intensity by unbound BSA, DLS size results are consistent with results obtained after fractionation by AFFF. Additionally, the AuNP hydrodynamic size exhibits a greater proportional increase due to BSA conjugation at $\mathrm{pH}$ values below 2.5 compared with less acidic $\mathrm{pH}$ values (3.4-7.3), corresponding with the reversibly denatured ( $\mathrm{E}$ or $\mathrm{F}$ form) conformation of $\mathrm{BSA}$ below $\mathrm{pH}$ 2.5. Over the $\mathrm{pH}$ range from 3.4 to 7.3 , the hydrodynamic size of the conjugate is nearly constant, suggesting conformational stability over this range. Because of the difference in the measurement environment, a larger increase of AuNP size is observed following BSA conjugation when measured in the wet state (i.e., by DLS and AFFF) compared to the dry state (by ES-DMA). Molecular surface density for BSA is estimated based on ES-DMA and fluorescence measurements. Results from the two techniques are consistent and similar, but slightly higher for ES-DMA, with an average adsorbate density of $0.015 \mathrm{~nm}^{-2}$. Moreover, from the change of particle size, we determine the extent of adsorption for BSA on AuNPs using DLS and ES-DMA at $21^{\circ} \mathrm{C}$, which show that increasing the concentration of BSA increases the measured change in AuNP size. Using ES-DMA, we observe that the BSA surface density reaches $90 \%$ of saturation at a solution phase concentration between 10 and $30 \mu \mathrm{mol} / \mathrm{L}$, which is roughly consistent with fluorescence and ATR-FTIR results. The equilibrium binding constant for BSA on AuNPs is calculated by applying the Langmuir equation, with resulting values ranging from $0.51 \times 10^{6}$ to $1.65 \times 10^{6} \mathrm{~L} / \mathrm{mol}$, suggesting a strong affinity due to bonding between the single free exterior thiol on $\mathrm{N}$-form BSA (associated with a cysteine residue) and the AuNP surface. Moreover, the adsorption interaction induces a conformational change in BSA secondary structure, resulting in less $\alpha$-helix content and more open structures $(\beta$-sheet, random, or expanded).

\section{INTRODUCTION}

The gold nanoparticle-protein conjugate, in which the protein is physically or chemically bound to the surface of a gold nanoparticle (AuNP), has attracted substantial interest in the areas of biodiagnostics and targeted drug delivery for cancer therapeutics. ${ }^{2-5}$ The formation of protein conjugates allows for the stabilization of AuNPs over a broad range of $\mathrm{pH}$ and ionic strengths and enables the introduction of biologically active functionalities either directly (as part of the protein itself) or via further couplings with the surface-bound protein or underlying gold surface. ${ }^{6,7}$ For example, antibodyfunctionalized AuNPs and tumor necrosis factor (TNF)conjugated AuNPs ${ }^{3-5}$ have been used for targeted cancer therapeutics.

Received: October 13, 2010

Revised: December 14, 2010

Published: February 22, 2011 

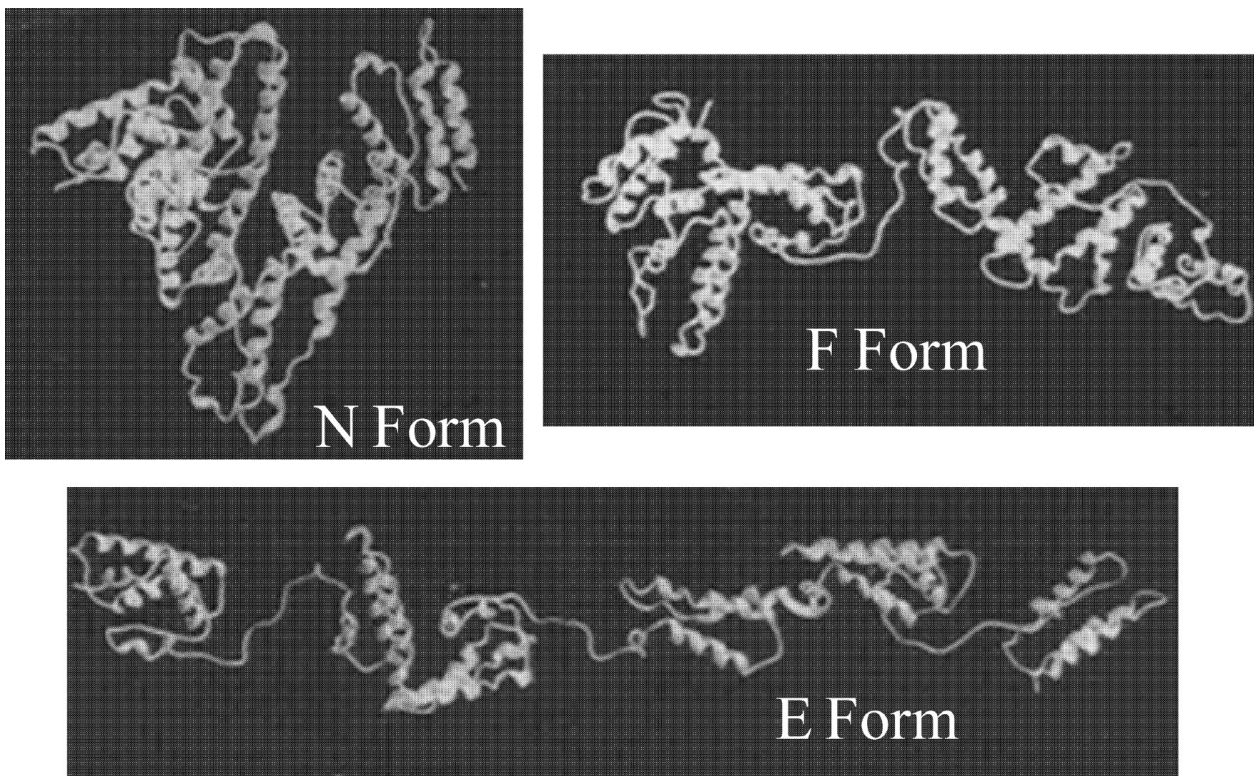

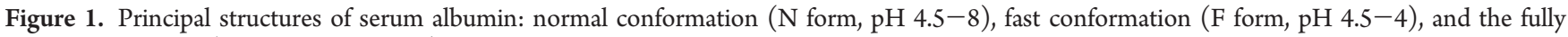
extended structure (E form, below $\mathrm{pH} 4$ ). Reproduced with permission from ref 12. Copyright 1994 Elsevier.

Furthermore, upon introduction of nanoparticles into living systems, a protein corona is formed, as serum proteins dynamically associate with the particle surface. ${ }^{9,10}$ The presentation of the serum proteins on the particle surface determines the subsequent in vivo response and substantially modifies the nanoparticle dimensions and transport properties, as demonstrated by Dobrovolskaia et al. for AuNPs. ${ }^{11}$ Therefore, understanding the interaction between AuNPs and proteins is vitally important for understanding how nanoparticles interact and behave in biological systems. ${ }^{9,10}$ This is particularly true for nanomedicine applications, where the regulatory approval required for clinical use sets a high standard for proof of efficacy and safety.

Among the various classes of proteins, albumin (or serum albumin) is conspicuous; it is the most abundant protein in blood, where it is responsible for about $80 \%$ of the colloidal osmotic pressure. ${ }^{12,13}$ As a multifunctional transport protein, albumin has a broad affinity for many ligands; for instance, albumin acts as a carrier for otherwise insoluble fatty acids in the circulatory system. ${ }^{14}$ In addition, albumin plays an important role in the transport and deposition of a variety of substances in therapeutic studies. ${ }^{12,15-17}$ Albumin is also chiefly responsible for regulating blood $\mathrm{pH}^{18}$ Recently, Porter and co-workers ${ }^{19,20}$ proposed using series-specific albumin in combination with a naturally occurring lung fluid surfactant dissolved in phosphate buffered saline (PBS) as a dispersion medium for nanoparticles in the context of in vivo pulmonary toxicity testing. Additional studies have established the general efficacy of albumin proteins as dispersing agents for a range of nanomaterials in different biological media. ${ }^{21-24}$ Furthermore, albumin continues to be used as a model protein for many biophysical and biochemical studies. $^{6,25-27}$

Bovine serum albumin (BSA) is the most widely utilized serum protein due to its low cost, wide availability, and structural/ functional similarity to human serum albumin (HSA) - having $76 \%$ sequence homology ${ }^{28}$ and nearly identical pH-dependent conformational transitions. ${ }^{29} \mathrm{BSA}$ is a water-soluble monomeric protein with a primary structure containing a single polypeptide chain with 583 amino acid residues and a molar mass of 66.4 $\mathrm{kDa}^{30}$ The secondary structure purportedly contains roughly $67 \% \alpha$-helix structure with the remainder being random. ${ }^{12}$ The tertiary structure consists of nine loops stabilized by 17 internal disulfide bonds between 34 cysteine residues, resulting in three primary domains each containing one small and two large loops. ${ }^{31}$ These disulfide bridges are the basis for the compact heart-shaped (equilateral triangle) structure (the so-called $\mathrm{N}$ form; see Figure 1$)^{12}$ now proposed for BSA in the $\mathrm{pH}$ range from 4.5 to 8 as well as providing stability to the helical structure. $^{31,32}$ The $\mathrm{N}$ form of BSA is characterized by an axial ratio of 2.7, a molecular volume of about $88.25 \mathrm{~nm}^{3}$, and a radius of gyration or hydrodynamic radius of about $2.6 \mathrm{~nm}^{33}$ and is slightly negatively charged (though the charge is heterogeneously distributed across the domains). ${ }^{28}$ Below $\mathrm{pH} 4.5$, a reversible unfolding occurs that results in the so-called $\mathrm{F}$ form, which is characterized by a more elongated shape (roughly $4 \mathrm{~nm} \times 12.9 \mathrm{~nm}$ ), reduced solubility, and increased viscosity. ${ }^{34,35}$ Below $\mathrm{pH} 4$, an additional transition takes place resulting in a fully extended structure (the $\mathrm{E}$ form), which is characterized by an aspect ratio of $9 .{ }^{36}$ In the $\mathrm{N}$ form, only a single free thiol (Cys-34) is accessible on the BSA surface, whereas in the F and $E$ forms, the normally inaccessible disulfides may become accessible to reducing agents or coupling with the AuNP surface. ${ }^{37}$ The Cys-34 thiol group is also responsible for the occurrence of dimers in aged BSA solutions. ${ }^{28}$ At $\mathrm{pH}$ values above 8 , another transition occurs resulting in the basic or $\mathrm{B}$ form. ${ }^{12,28}$

Even with all that is currently known about native BSA, detailed information on BSA-AuNP conjugates is relatively sparse. ${ }^{6,7,9,15,38-43}$ Additionally, a few related studies have examined the adsorption and conformation of BSA onto Au flats. ${ }^{17,44,45}$ The structure and behavior of BSA in aqueous solution are governed by multiple interactions, including electrostatic, hydrogen bonding, hydrophobic, and van der Waals. In addition, the reaction of the free thiol on the external surface of 
globular ( $\mathrm{N}$ form) BSA with the AuNP surface could potentially weaken or alter the tertiary or secondary structure of the BSA molecule, which would likely result in the unfolding of BSA from its compact, globular structure. ${ }^{46}$ Hence, after binding to AuNPs, BSA may undergo structural changes at the interface, and the resulting less-compact structure may affect BSA's function and bioactivity as well as the stability, reactivity, and transport properties of the AuNP-BSA conjugate. To understand the interactions between BSA and AuNPs, it is therefore necessary to conduct a quantitative investigation requiring complementary and orthogonal characterization techniques. The objective here is to interrogate structural changes in BSA when it interacts with the AuNP surface, to determine BSA surface density, to measure the binding affinity, and finally to determine how the solution environment influences these factors.

Physical characterization methods, such as dynamic light scattering (DLS) and electrospray-differential mobility analysis (ES-DMA), have been utilized in previous studies for the direct characterization of molecular conjugation to nanoparticles. ${ }^{47-50}$ The general concept is to measure the increase in particle diameter as a result of ligand adsorption on the AuNP surface. Surface density of conjugated molecules is quantified based on the increase in particle size attributable to the ligands, after accounting for other effects. ${ }^{4-50}$ Through the use of an analytical model, the surface density and molecular conformation can be estimated based on the calculated molecular size for different surface densities and measurement environments.

Although physical characterization methods have demonstrated a facile capacity to characterize molecular conjugation on nanoparticles with high speed and resolution, ${ }^{49-51}$ an obvious capability gap is that particle size is the only experimental output. For the study of competitive adsorption between two molecules, it is a challenge to differentiate the individual contributions to the particle size arising from each species. In addition, for nanoparticles having a broad size distribution, it is difficult to determine if the increase in particle size is due to molecular conjugation or particle aggregation. Spectroscopic methods ${ }^{52-54}$ provide an orthogonal comparison to these physical characterization approaches. By monitoring the change in optical signals corresponding specifically to BSA (absorption or fluorescence), it is possible to track the change in surface density of BSA on AuNPs. One advantage of using spectroscopic methods is to confirm that observed changes in particle size measured by physical methods are in fact due to BSA conjugation and not to particle aggregation. Also, spectroscopic methods provide chemical specificity that allows the study of multipleadsorbate systems, even when the adsorbates may have a similar physical size.

In the present work, we use DLS to determine particle size and size distribution as a starting point due to the wide availability of this technique. ${ }^{50,55}$ DLS is then benchmarked using fractionation techniques: asymmetric-flow field flow fractionation (AFFF) in the wet state and ES-DMA in the dry (aerosol) state. By characterizing the particle size and size distribution using these complementary physical methods, we can probe the formation of BSA surface coatings and analyze its conformation on AuNPs under different environmental conditions. For comparison with the physical characterization methods, fluorescence assay and attenuated total reflectance-Fourier transform infrared (ATRFTIR) spectroscopy are used to quantify adsorption density of BSA on AuNPs and to evaluate the change in BSA conformation following conjugation.

\section{EXPERIMENTAL SECTION}

2.1. Materials. Commercially available, 10,30 , and $60 \mathrm{~nm}$ citratestabilized monodisperse colloidal AuNPs were obtained from Ted Pella Inc. (Redding, CA). ${ }^{56}$ The number concentration of particles in solution is estimated to be $5 \times 10^{12}, 2 \times 10^{11}$, and $2.6 \times 10^{10} \mathrm{~cm}^{-3}$ for nominal 10, 30, and $60 \mathrm{~nm}$ AuNPs, respectively. The volumetric ratio (i.e., the volume ratio of AuNP to water) is estimated as $3 \times 10^{-6}$ for all three nominal sized AuNPs. Highly purified reagent-grade BSA ( $\geq 98 \%$ protein, $\leq 3 \mathrm{EU} / \mathrm{mg}$ endotoxin, fatty acid- and IgG-free, SeraCare Life Science, Milford, MA) was utilized at concentrations ranging from 1.6 to $100 \mu \mathrm{mol} / \mathrm{L}$. For sample preparation of BSA-conjugated AuNPs, $200 \mu \mathrm{L}$ of BSA aqueous solutions $(0.2-1000 \mu \mathrm{mol} / \mathrm{L})$ were first prepared and then mixed with $800 \mu \mathrm{L}$ of AuNPs. Aqueous ammonium acetate (99.9\%, Sigma-Aldrich, St. Louis, MO) solution was prepared to adjust the ionic strength $(2.5 \mathrm{mmol} / \mathrm{L}, 0.03 \mathrm{~S} / \mathrm{m})$. pH values were measured using an Orion 3 STAR meter (Thermo Electron, Madison, WI) with a InLab semi-microelectrode (Mettler, Teledo, MI). Biological grade 18.2 $\mathrm{M} \Omega \cdot \mathrm{cm}$ deionized water (Aqua Solutions, Jasper, GA) was used for preparing solutions and AuNP suspensions with various concentration factors.

2.2. Dynamic Light Scattering. DLS measurements were performed using a Zetasizer Nano (Malvern Instruments, Westborough, MA). Details of the DLS experimental setup and analysis have been described in previous publications. ${ }^{47,50}$ We employ the cumulants method to determine the mean $z$-average hydrodynamic diameter, $d_{\mathrm{p}, \mathrm{hz}}$, and the polydispersity index (PI); for additional information and experimental details refer to section 8.1 of the Supporting Information. The DLS instrument used in this study was operated in both batch and online (flow) mode, the latter in conjunction with AFFF fractionation (see below).

2.3. Electrospray-Differential Mobility Analysis (ES-DMA). The ES-DMA system consists of an electrospray aerosol generator (model 3480, TSI Inc., MN), a differential mobility analyzer (DMA, model 3080n, TSI Inc.), and a condensation particle counter (CPC, model 3025, TSI Inc.). Details of the ES-DMA experimental setup have been described in previous publications. ${ }^{48,49,54,57-59}$ Additional experimental details are summarized in section 8.2 of the Supporting Information.

2.4. Asymmetric-Flow Field Flow Fractionation (AFFF). The AFFF system consists of a high-performance liquid chromatography (HPLC) isocratic pump (1100 series, Agilent Technologies, Santa Clara, CA), manual injection valve (Rheodyne 7725i, IDEX Corporation, Oak Harbor, WA) with a $100 \mu \mathrm{L}$ stainless steel sample loop, field/flow control module, and AFFF separation channel (Eclipse 2, Wyatt Technology, Santa Barbara, CA), multiangle light scattering (MALS) detector (Dawn Heleos, Wyatt Technology), and ultraviolet-visible (UV-vis) absorbance diode array detector (1200 DAD, Agilent Technologies). The instrument configuration uses a DLS device operated in flow mode as the final detector (Zetasizer Nano, Malvern Instruments Inc.). Details of the AFFF experimental setup have been described in a previous publication. ${ }^{60}$ Finally, the particle size corresponding to an eluting peak can be calibrated in situ by flow mode DLS measurements. For a summary of the method and additional experimental details, refer to section 8.3 of the Supporting Information.

2.5. Attenuated Total Reflectance-Fourier Transform Infrared (ATR-FTIR) Spectroscopy. ATR-FTIR spectroscopy was performed using a Nicolet Spectra 750 FTIR equipped with a Thunder Dome Germanium ATR accessory (Thermo Scientific, Madison, WI). Spectra were collected from an average of 128 scans with a resolution of $1 \mathrm{~cm}^{-1}$. For sample preparation, $800 \mu \mathrm{L}$ of as-received AuNP solution was first concentrated $16 \times$ by centrifugation and then drop-cast onto the surface of the germanium crystal. After evaporating the solvent in a clean bench at room temperature $\left(21^{\circ} \mathrm{C}\right)$, a AuNP film 
was formed on the crystal surface (roughly $300 \mathrm{~nm}$ in thickness). Then, a flow cell unit (Thermo Scientific) was attached to the top of the crystal in order to allow fluid to contact and penetrate the AuNP film. The background spectra were recorded by introducing deionized (DI) water into the flow cell. BSA solutions under various experiment conditions (e.g., $\mathrm{pH}, \mathrm{BSA}$ concentration) were then introduced into the flow cell, and the resulting spectra were recorded.

2.6. Fluorescence Spectrometry. Fluorescence spectrometry was conducted using a Quant-iT protein assay kit (Invitrogen, Paisley, UK). The assay involves a dye that fluoresces when bound to the detergent coating and/or hydrophobic regions of proteins, while the unbound dye is not fluorescent. First, $200 \mu \mathrm{L}$ of the working solution, containing the fluorescent dye and the protein buffer, was loaded into each microplate well, and then 5-20 $\mu \mathrm{L}$ test samples of the AuNP-BSA were added into each well so that the fluorophore could bind to the protein. BSA standards $(10 \mu \mathrm{L})$ with concentrations in the range $25-$ $500 \mathrm{ng} / \mu \mathrm{L}$ were placed in separate wells to facilitate quantitative calibration. A SAFIRE multidetection monochromator microplate reader (Tecan Inc., Durham, NC) was employed for the fluorescence measurements. The excitation wavelength was $470 \mathrm{~nm}$, and the fluorescence was measured at $570 \mathrm{~nm}$.

Through use of calibration with standards, the amount of BSA presenting in AuNP samples can be quantified based on the corresponding fluorescence intensity, $I_{\mathrm{f}}$. For the concentration range we cover, the amount of BSA presenting in each well can be calculated using a correlation of $\left(I_{\mathrm{f}}+28\right) / 0.4833$ (expressed as $10^{-9} \mathrm{~g}$ of BSA).

2.7. Measurement Uncertainty. Error bars shown in figures and uncertainty ranges associated with measurement values represent one standard deviation calculated from replicate $(2-4)$ measurements performed under repeatability conditions.

\section{RESULTS AND DISCUSSION}

3.1. Physical Characterization of BSA Conjugation on AuNPs in the Wet State. DLS was used to characterize the hydrodynamic size, $d_{\mathrm{p}, \mathrm{hz}}$, of AuNPs before and after BSA conjugation. The non-negative least-squares (NNLS) regularization algorithm has been applied widely for calculating particle size distributions from DLS data (Figure S1, Supporting Information). However, because of the lack of standardization for NNLS and other inversion algorithms, the cumulants approach was chosen for the analysis of DLS data in this study. ${ }^{61,62}$

As shown in Figure 2a for nominally $10 \mathrm{~nm}$ AuNPs, following conjugation with BSA the correlation function, $G(\tau)$, exhibits a nonlinear decay (i.e., a significant secondary coefficient $\mu_{2}$ is evident); this indicates an increase in the variance of particle diffusion coefficients (or PI) relative to the native particles and suggests increased polydispersity in size. When the nominal (core) size is increased to $60 \mathrm{~nm}$ (Figure 2b), the correlation decay becomes nearly linear (i.e., $\mu_{2}$ decreases with increasing particle size), indicating that the PI does not increase following BSA conjugation and suggesting an absence of agglomeration. From the first-order coefficient, $\bar{\Gamma}$, obtained from data shown in Figure $2 \mathrm{a}, \mathrm{b}, d_{\mathrm{p}, \mathrm{hz}}$ was calculated. Here the amount of BSA presenting in solution is at least 1000 times higher than the required amount to have a maximum monolayer surface coverage. $^{6,27,63}$ Figure $2 \mathrm{c}$ summarizes the change in $d_{\mathrm{p}, \mathrm{hz}}$ (denoted $\Delta d_{\mathrm{p}, \mathrm{hz}}$ ) and PI (denoted $\Delta \mathrm{PI}$ ) as a function of the native (core) diameter. As the core size increases, $\Delta d_{\mathrm{p}, \mathrm{hz}}$ increases while $\Delta$ PI decreases. Note also that $\Delta d_{\mathrm{p}, \mathrm{hz}}$ is close to zero for the smallest core size, even though the PI increases following conjugation. If agglomeration were occurring, both $\Delta d_{\mathrm{p}, \mathrm{hz}}$ and PI should increase. Similarly, if conjugation were occurring in a heterogeneous manner (i.e., more BSA adsorption on some particles and less on others), both $\Delta d_{\mathrm{p}, \mathrm{hz}}$ and PI should increase, though relatively less so than would be expected for agglomeration effects. We have also observed that $\Delta d_{\mathrm{p}, \mathrm{hz}}$ exhibits a dependence on the concentration factor (i.e., the number concentration of AuNPs used in experiments compared to the as-received particle concentration). As shown in Figure $2 \mathrm{~d}$, a negative $\Delta d_{\mathrm{p}, \mathrm{hz}}(\approx-1$ to $-5 \mathrm{~nm})$ was observed for nominally 10 $\mathrm{nm}$ AuNPs at all dilution levels; the variation in the measurement results versus concentration factor can be attributed primarily to the fitting uncertainty in the autocorrelation function (Supporting Information, eq S1). For nominally $30 \mathrm{~nm}$ AuNPs, increasing the concentration factor increases $\Delta d_{\mathrm{p}, \mathrm{hz}}$ significantly from -9.6 to $8.5 \mathrm{~nm}$. For nominally $60 \mathrm{~nm}$ AuNPs, increasing the concentration factor increases $\Delta d_{\mathrm{p}, \mathrm{hz}}$ from 7.3 to $11.4 \mathrm{~nm}$, which indicates that the concentration factor is less significant as compared to the two smaller size AuNPs. Similar to the results in Figure $2 c$, a larger $\Delta$ PI value was observed when $\Delta d_{\mathrm{p}, \mathrm{hz}}$ was close to zero or negative. Finally, it is important to note that the concentration factor does not have a significant impact on either $\Delta d_{\mathrm{p}, \mathrm{hz}}$ or $\Delta \mathrm{PI}$ for unconjugated AuNPs over the range studied here.

On the basis of the above arguments, we conclude that the dependencies on the core size and particle concentration factor results from the presence of unbound globular BSA molecules. The free BSA apparently contributes sufficiently to the measured correlation function to interfere with measurement of the AuNPs (i.e., convolution of scattering from both species impacts the cumulants determination of the mean $z$-average size); this effect is noticeable for AuNP core sizes as large as $30 \mathrm{~nm}$, whereas for the $60 \mathrm{~nm}$ cores the particle scattering dominates the correlation spectrum sufficiently and the effect of free BSA is insignificant.

The BSA interference can be analyzed assuming Rayleigh scattering properties, in which the scattering intensity is proportional to $N_{\mathrm{p}} d_{\mathrm{p}, \mathrm{hz}}{ }^{6}$. The hydrodynamic diameter of a BSA monomer, $d_{\mathrm{BSA}, \mathrm{hz}}$, has been reported or estimated to be about $4.2-$ $7 \mathrm{~nm},{ }^{31,51,64-66}$ which is comparable to the $10 \mathrm{~nm}$ AuNPs used in our experiments. In addition to $d_{\mathrm{BSA}, \mathrm{hz}}$, the number of free BSA molecules in solution, $N_{\mathrm{BSA}}$, is at least $10^{4}$ times the population of AuNPs, $N_{\mathrm{p}}$. Hence, the light scattering intensity from unbound BSA can be significant compared to the scattering intensity from AuNPs, especially for particles having smaller core size (i.e., $10 \mathrm{~nm}$ AuNPs). When $d_{\mathrm{p} 0}$ increases to $60 \mathrm{~nm}$, the artifact resulting from the free BSA is negligible.

To quantify the impact of the free BSA on the scattering measurement, we define a dimensionless parameter

$$
\gamma=\frac{\text { AuNP scattering intensity }}{\text { BSA scattering intensity }}=\frac{\varepsilon_{\mathrm{p}} N_{\mathrm{p}} d_{\mathrm{p} 0}{ }^{6}}{\varepsilon_{\mathrm{BSA}} N_{\mathrm{BSA}} d_{\mathrm{BSA}}{ }^{6}}
$$

where $\varepsilon_{\mathrm{p}}$ and $\varepsilon_{\mathrm{BSA}}$ are the refractive index for AuNPs and BSA, respectively. In this study, we used $\varepsilon_{\mathrm{p}}=0.47^{67}$ and $\varepsilon_{\mathrm{BSA}}=1.445$. $^{41}$ $d_{\mathrm{p} 0, \mathrm{hz}}$ is the hydrodynamic diameter for unconjugated Au-NPs. With an increase in $\gamma$, the impact from free BSA decreases, and therefore the accuracy of $\Delta d_{\mathrm{p}, \mathrm{hz}}$ improves. For example, the molar mass $(M)$ of $5 \mathrm{kDa}$ thiolated poly(ethylene glycol) (SHPEG5K) is $13 \times$ smaller than a BSA monomer; therefore, the scattering intensity from unbound SH-PEG5K should be $170 \times$ lower than that from BSA at the same solution concentration. Hence, the contribution from the unbound SH-PEG5K is negligible for AuNPs used in the previous study. ${ }^{50}$ 

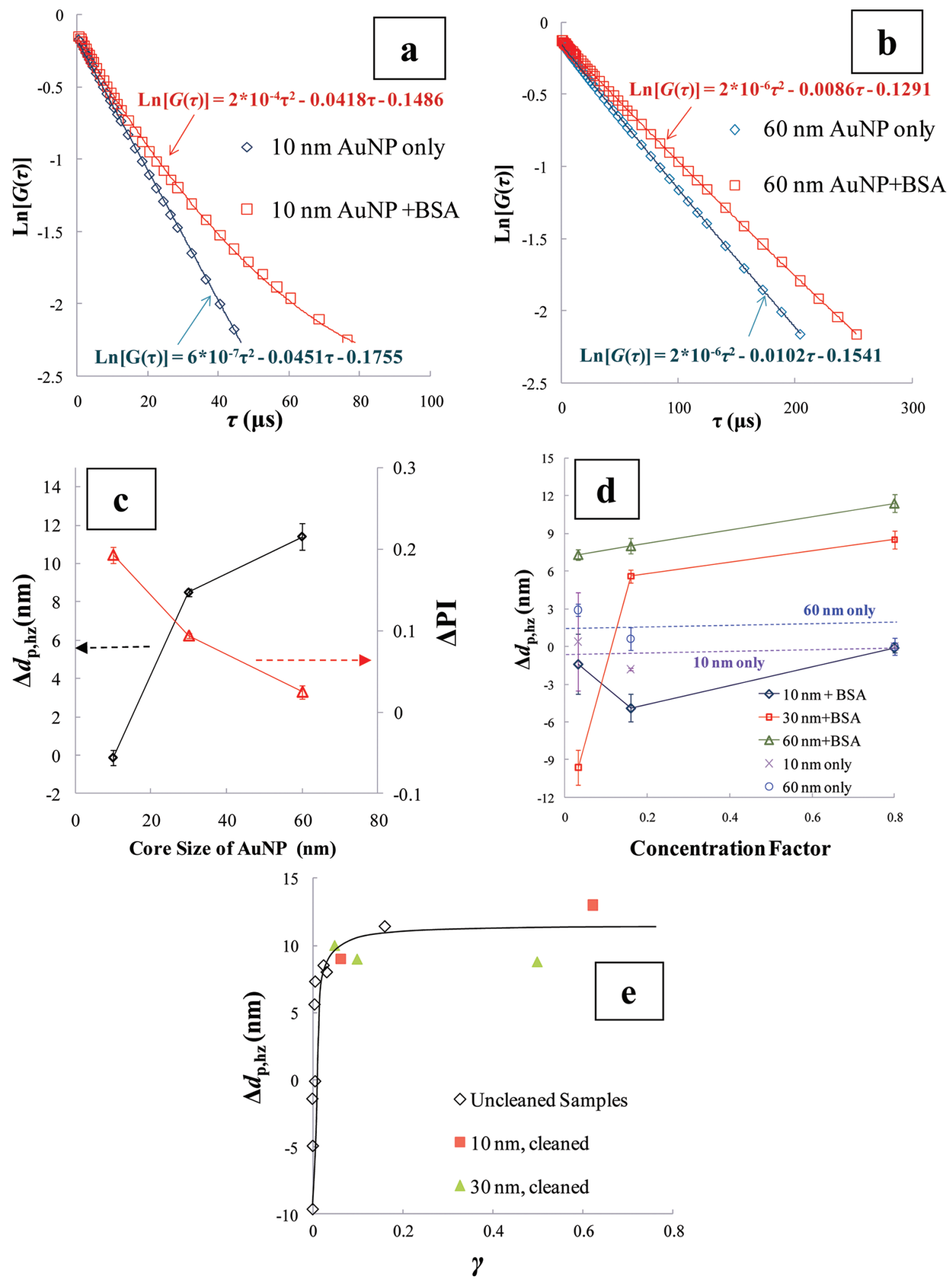

Figure 2. Characterization of BSA conjugation on AuNPs by DLS. (a) Cumulant analysis, $10 \mathrm{~nm}$ AuNPs with $100 \mu \mathrm{mol} / \mathrm{L}$ of BSA. (b) Cumulant analysis, $60 \mathrm{~nm}$ AuNPs with $100 \mu \mathrm{mol} / \mathrm{L}$ of BSA. (c) Effect of AuNP core size on $\Delta d_{\mathrm{p}, \mathrm{hz}}$ and $\Delta \mathrm{PI}$ without centrifuge treatment. Concentration of AuNPs is $80 \%$ of the as-received concentration (concentration factor $=0.8$ ). $C_{\mathrm{BSA}}$ is $100 \mu \mathrm{mol} / \mathrm{L}$. The solid line is to guide the eyes. (d) Effect of particle concentration on $\Delta d_{\mathrm{p}, \mathrm{hz}}$. Different concentration factors for AuNPs with $100 \mu \mathrm{mol} / \mathrm{L}$ of BSA. Two reference data sets $(10 \mathrm{and} 60 \mathrm{~nm}$ cores without BSA) were used to show $\Delta d_{\mathrm{p}, \mathrm{hz}}$ vs concentration factor without BSA conjugation. The solid line is to guide the eyes. (e) $\Delta d_{\mathrm{p}, \mathrm{hz}}$ versus $\gamma$; the centrifuged samples were at a concentration factor of 0.8 , and the uncentrifuged samples represent different nominal core sizes $(10,30$, and $60 \mathrm{~nm}$, open diamonds) and concentration factors. $\mathrm{pH}$ of samples ranged from 6.0 to 7.9. The solid line fit for open diamonds (no centrifugation) in (e) is to guide the eyes. Data points are the mean values of at least four replicate measurements, and error bars (smaller than the symbol size) represent one standard deviation. 
Table 1. Summary of the Change of Particle Size and $\Delta$ PI Measured by DLS and ES-DMA

\begin{tabular}{|c|c|c|c|c|c|c|c|}
\hline $\begin{array}{l}\text { nominal AuNP } \\
\text { size }(\mathrm{nm})\end{array}$ & $d_{\mathrm{p} 0, \mathrm{hz}}(\mathrm{nm})$ & $d_{\mathrm{p} 0, \mathrm{~m}}{ }^{a}(\mathrm{~nm})$ & $\begin{array}{l}\Delta d_{\mathrm{p}, \mathrm{hz}} \text {, with } \\
\mathrm{BSA}(\mathrm{nm})\end{array}$ & $\begin{array}{c}\Delta d_{\mathrm{p}, \mathrm{hz}} \text {, with BSA + } \\
\quad \text { cleaning }(\mathrm{nm})\end{array}$ & $\begin{array}{c}\Delta d_{\mathrm{p}, \mathrm{m}},{ }^{a} \text { with } \\
\text { BSA }(\mathrm{nm})\end{array}$ & $\begin{array}{l}\Delta \mathrm{PI} \text {, with } \\
\mathrm{BSA}(\mathrm{nm})\end{array}$ & $\begin{array}{l}\Delta \mathrm{PI} \text {, with BSA after } \\
\text { cleaning }(\mathrm{nm})\end{array}$ \\
\hline 10 & $13.8 \pm 0.5$ & $13.0 \pm 0.1$ & $-2.0 \pm 2.5$ & $13.2 \pm 0.2$ & $6.3 \pm 0.1$ & $0.193 \pm 0.01$ & $-0.089 \pm 0.043$ \\
\hline 30 & $30.4 \pm 0.1$ & $31.1 \pm 0.3$ & $8.5 \pm 0.7$ & $8.3 \pm 0.2$ & $6.1 \pm 0.3$ & $0.094 \pm 0.003$ & $0.036 \pm 0.015$ \\
\hline 60 & $58.2 \pm 0.4$ & $59.0 \pm 0.3$ & $11.4 \pm 0.7$ & $11.5 \pm 1.1$ & $5.9 \pm 0.3$ & $0.025 \pm 0.008$ & $0.012 \pm 0.013$ \\
\hline
\end{tabular}

Figure 2e summarizes the correlation between $\Delta d_{\mathrm{p}, \mathrm{hz}}$ and $\gamma$. The role of $\gamma$ in Figure 2e is to serve as an indicator with regards to selecting suitable conditions for conducting DLS measurements in the AuNP-BSA system. The $\gamma$ values of the centrifuged samples are calculated based on the amount of supernatant (containing excess BSA) removed in the centrifugation process, assuming no particle loss during centrifugation (e.g.: $\gamma$ is increased $20 \times$ if replacing $950 \mu \mathrm{L}$ supernatant from $1000 \mu \mathrm{L}$ of Au colloids). The open diamond symbols represent the data of precentrifuged samples for three different core size Au-NPs, whereas the solid line fitted through the open diamonds is used to guide the eyes. Clearly, $\Delta d_{\mathrm{p}, \mathrm{hz}}$ reaches a plateau when $\gamma>0.1$; in order to mitigate the artifact from the unbound BSA molecules, $\gamma$ should be at least 0.1 . For smaller AuNPs with a lower particle concentration, removing the unbound BSA molecules by centrifugation and supernatant replacement can improve $\gamma$. As shown in Figure 2e, using the centrifugation approach, $\Delta d_{\mathrm{p}, \mathrm{hz}}$ for both 10 and $30 \mathrm{~nm}$ AuNPs increases and becomes nearly coincident with $\Delta d_{\mathrm{p}, \mathrm{hz}}$ for $60 \mathrm{~nm}$ AuNPs that have not been centrifuged: $\Delta d_{\mathrm{p}, \mathrm{hz}}$ is 13.2 and $8.3 \mathrm{~nm}$ for 10 and $30 \mathrm{~nm}$, respectively. Table 1 summarizes the change of particle size and $\triangle P I$ of AuNPs before and after conjugating with BSA. Note that the negative values for $\Delta d_{\mathrm{p}, \mathrm{hz}}$ observed in Figure $2 \mathrm{c}, \mathrm{d}$ arise from an artifact of the cumulants analysis. In this case, the contribution to the correlation decay associated with the unbound BSA is averaged with the principal correlation decay of the BSA-conjugated AuNPs leading to an apparent decrease in size for the AuNPs. This artifact is eliminated when the unbound BSA is removed, for instance, by centrifugation.

In order to confirm $\Delta d_{\mathrm{p}, \mathrm{hz}}$ values determined by batch-mode DLS and to rule out other potential effects such as agglomeration, we employed AFFF to fractionate test samples prior to online analysis. In addition to fractionation and size determination (based on retention time), on-board UV-vis absorption spectroscopy, multiangle light scattering (MALS), and flow-mode DLS detectors permit differentiation of components by size and chemical speciation. In Figure 3a, nominally $60 \mathrm{~nm}$ AuNPs were chosen to exemplify the method, though it should be applicable to other particle sizes, including nominally 10 and $30 \mathrm{~nm}$ AuNPs. ${ }^{60}$ After converting retention time to equivalent spherical hydrodynamic size, the fractograms measured by AFFF for nominally $60 \mathrm{~nm}$ AuNPs, with and without BSA, are shown in Figure 3a. The corresponding UV-vis spectra obtained at the peak positions denoted 1 to 4 in Figure 3a are shown in Figure $3 b$. Note that the concentration factor used for AFFF measurements was 0.08 for $60 \mathrm{~nm}$ AuNPs.

Three modes (peaks) were observed in the MALS trace (measured at $90^{\circ}$ ) for BSA-conjugated AuNPs, which contain both bound and free BSA. This trace should be compared with the MALS trace for native (preconjugation) AuNPs, also shown in Figure 3a. The small mode identified as peak 1 in Figure 3a, located at $\approx 5 \mathrm{~nm}\left(t_{\mathrm{r}}-t_{0}=2.9 \mathrm{~min}\right.$, void peak at $t_{0}$ is not shown $)$
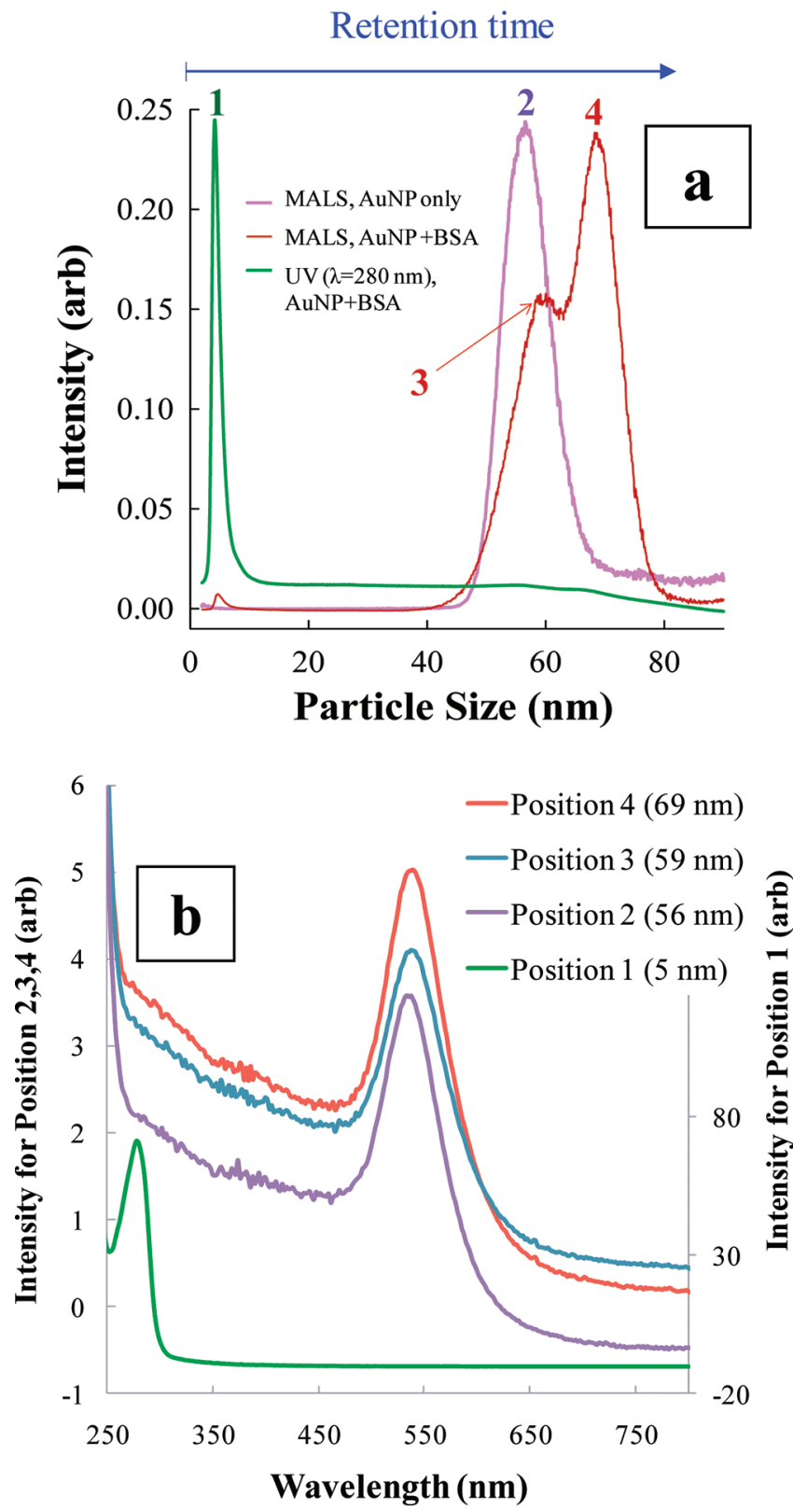

Figure 3. Characterization of BSA conjugation on nominal $60 \mathrm{~nm}$ AuNPs using AFFF. (a) MALS and UV $(\lambda=280 \mathrm{~nm})$ traces for AuNPs. (b) UV-vis spectra obtained online at specific points identified in (a). $C_{\mathrm{BSA}}$ is $100 \mu \mathrm{mol} / \mathrm{L}$, the concentration factor is 0.08 , and $\mathrm{pH}$ is 6.7 . Void peaks are not shown in the figure.

in both the UV trace and the MALS trace, is attributed to unbound BSA (the maximum absorption wavelength, $\lambda_{\max }$ occurs at $276 \mathrm{~nm}$ ). The peaks located between 59 and $67 \mathrm{~nm}$ 
(peak 2 for native AuNPs, peaks 3 and 4 for BSA-AuNPs) are assigned to AuNPs; these peaks eluted from $t_{\mathrm{r}}-t_{0}=31$ to 37 min, respectively. The corresponding $\mathrm{UV}-$ vis spectra (Figure $3 \mathrm{~b}$ ) for peaks $2-4$ exhibit classic surface plasmon resonance bands for AuNPs in this size range $\left(\lambda_{\max } \approx 530\right.$ $\mathrm{nm})$. Moreover, although the MALS trace for BSA-conjugated AuNPs shows a doublet (peaks 3 and 4 in Figure 3a), the corresponding UV-vis spectra (with $\lambda_{\max } \approx 530 \mathrm{~nm}$ ), as well as online DLS measurements $\left(d_{\mathrm{p}, \mathrm{hz}} \approx 66 \mathrm{~nm}\right.$ as measured by Malvern Nano ZetaSizer, not shown in Figure 3), are virtually identical for peaks 3 and 4, suggesting these features represent a single species that is $66 \mathrm{~nm}$ in diameter (i.e., BSA-AuNPs). We attribute the peak splitting phenomenon to particle-membrane interactions, which are beyond the scope of the present work. However, this serves to emphasize the importance of combining multiple sensing or detection methods in order to accurately identify and characterize complex systems that may contain or exhibit multiple components, whether real or artifact. The increase in particle size, $\Delta d_{\mathrm{p}, \mathrm{hz}}$, based upon results from the online DLS is $9.3 \pm 0.3 \mathrm{~nm}$, which is close to the value obtained in batch mode DLS (see Figure 2). This confirms the homogeneous BSA conjugation to the surface of AuNPs.

3.2. Effect of $\mathrm{pH}$ on the Molecular Conformation of Adsorbed BSA. As stated previously (Figure 1), BSA undergoes conformational expansion from the heart-shaped $\mathrm{N}$ form (see Figure 1) that is associated with $\mathrm{pH}$ values above its isoelectric point $(\approx 4.7)$ to more elongated and open structures ( $\mathrm{F}$ and $\mathrm{E}$ forms) at $\mathrm{pH}$ values below its isoelectric point. ${ }^{12,32}$ Since a wide range of $\mathrm{pH}$ values may be encountered in biological systems (e.g., ranging from about $\mathrm{pH} 1.5$ to 3.5 in the human stomach to $\mathrm{pH} 4.7$ in lysosomes and $\mathrm{pH} 7.4$ in human blood), it is clearly important to understand the effect this will have on the structure and stability of surface-bound BSA, as this will likely impact AuNP conjugate properties and interactions. Presently, this area remains largely unexplored, ${ }^{25,68}$ especially in the use of direct physical characterization of surface-bound BSA. Much more work has been reported regarding the conformation of free (unbound) BSA. ${ }^{12,28,31,34,36,46,69}$ Relevant to the present study, Kun et al. ${ }^{69}$ employed DLS over various $\mathrm{pH}$ conditions and were able to demonstrate a larger hydrodynamic size for the $\mathrm{E}$ form than the $\mathrm{N}$ form. $\mathrm{Li}$ et $\mathrm{al}^{70}$ examined the conformation of BSA as a function of $\mathrm{pH}$ adsorbed at a flat gold surface using force microscopy and small-angle neutron scattering; their findings indicated that the conformation of BSA conjugates is more compact when the $\mathrm{pH}$ is close to the isoelectric point of native BSA (4.7). ${ }^{12,28}$ To provide direct information on the conformation of BSA adsorbed to AuNPs, we will utilize DLS to characterize the change of hydrodynamic size of the BSA conjugates over a broad range of $\mathrm{pH}$ values.

In order to simplify measurement conditions, experiments were conducted using nominally $60 \mathrm{~nm}$ AuNPs, since the high $\gamma$ value precludes having to remove the unbound BSA prior to DLS measurements. First, eight samples of BSA conjugated AuNPs were prepared at $\mathrm{pH} 7.3, C_{\mathrm{BSA}}=10 \mu \mathrm{mol} / \mathrm{L}$. Then these samples were titrated with nitric acid to their targeted $\mathrm{pH}$ values and measured by DLS within $1 \mathrm{~h}$ after $\mathrm{pH}$ adjustment. DLS results for the BSA-AuNP conjugates as a function of solution $\mathrm{pH}$ are shown in Figure 4a. Size is represented as the change in half of the hydrodynamic diameter, $0.5 \Delta d_{\mathrm{p}, \mathrm{hz}}$ (i.e., the presumed shell thickness), relative to the unconjugated AuNPs (determined under stable conditions near $\mathrm{pH} 7$ ). The results indicate a single sharp transition between two stable size regimes, while the PI remains relatively unchanged over the entire $\mathrm{pH}$ range tested
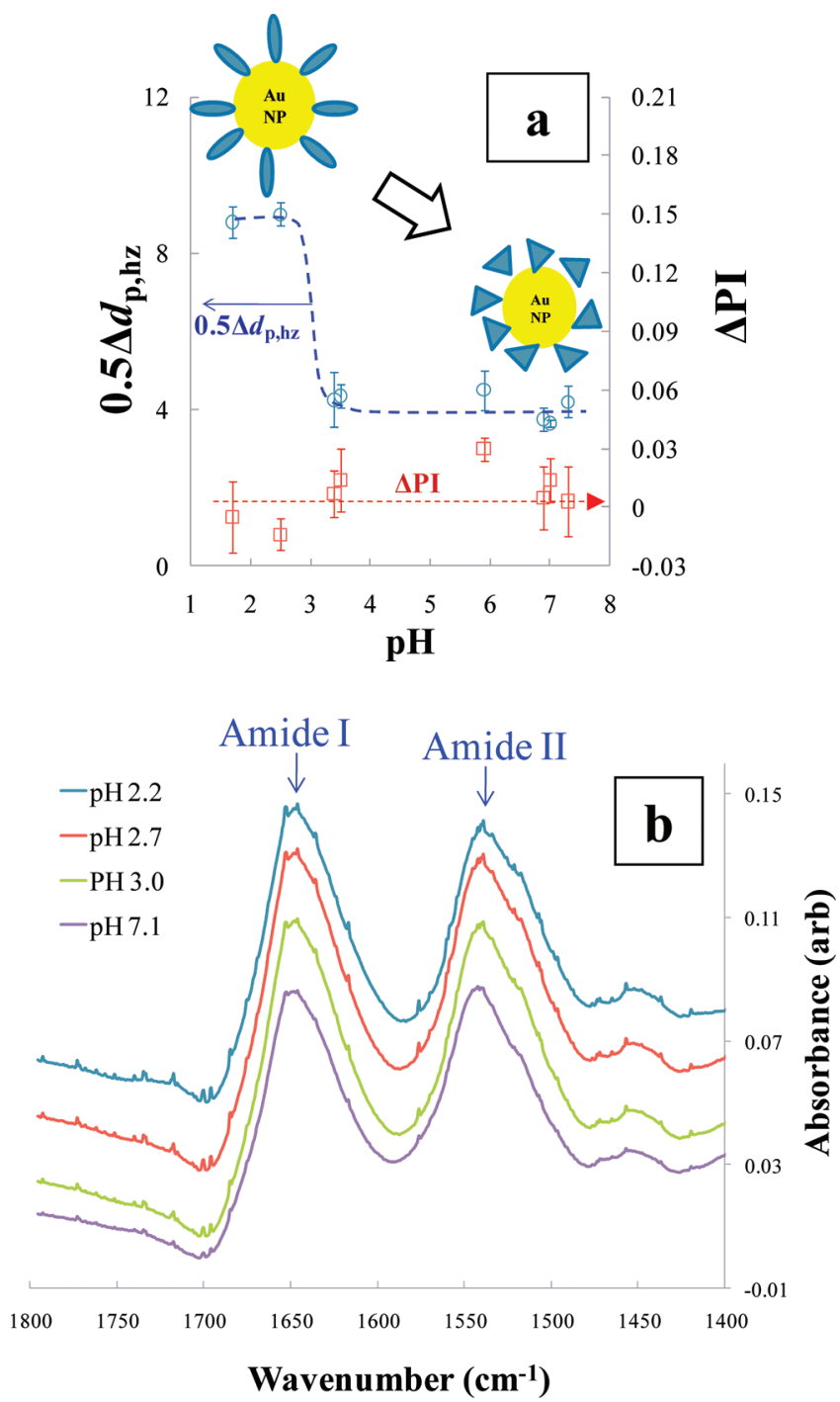

Figure 4. Effect of $\mathrm{pH}$ on the surface conformation of BSA conjugates on nominal $60 \mathrm{~nm}$ AuNPs. (a) $0.5 \Delta d_{\mathrm{p}, \mathrm{hz}}$ and $\Delta \mathrm{PI}$ as a function of $\mathrm{pH}$. Data were recorded within $1 \mathrm{~h}$ of adjusting $\mathrm{pH}$. Note that $\mathrm{pH}$ values were measured directly on the DLS samples. $C_{\mathrm{BSA}}$ is $10 \mu \mathrm{mol} / \mathrm{L}$. Data points are the mean values of four measurements, and error bars are one standard deviation. Lines are included for visual guidance. (b) ATRFTIR spectra for BSA-AuNP conjugates as a function of decreasing $\mathrm{pH}$ (from bottom to top). $C_{\mathrm{BSA}}$ is $10 \mu \mathrm{mol} / \mathrm{L}$. Spectra are vertically shifted for the purpose of clarity.

(ruling out agglomeration effects, but not multilayer adsorption). Below about pH 2.7 a thicker or more vertically aligned layer of BSA increases the hydrodynamic size of the conjugate, while above $\mathrm{pH} 3.4$ and up to physiological $\mathrm{pH}$ the hydrodynamic size remains stable and relatively smaller. The results are consistent with a transition between the $\mathrm{N}$ form and either the $\mathrm{F}$ or $\mathrm{E}$ form of free BSA but also suggest some conformational inhibition (i.e., lower transition $\mathrm{pH}$ ) relative to free BSA due to attachment on the Au surface; the $\mathrm{N}-\mathrm{F}$ transition for free BSA occurs above $\mathrm{pH}$ 4. So it is plausible that conjugated BSA exhibits only a single conformational transition, though we may simply not be able to resolve the transition from $\mathrm{F}$ to $\mathrm{E}$ based on DLS measurements alone. Another possibility is that the surface binding process between BSA and Au-NPs may itself induce conformation 
changes, similar to the $\mathrm{N}-\mathrm{F}$ transformation, even in the so-called $\mathrm{N}$ form $\mathrm{pH}$ regime. ${ }^{12,25}$ Hence, the transition between $\mathrm{N}$ and $\mathrm{F}$ forms becomes less observable.

To rule out the effect of possible differences in surface density or the occurrence of multilayers, we evaluated the effect of $\mathrm{pH}$ on BSA adsorption using ATR-FTIR. This analytical approach is surface sensitive due to the limited penetration of the evanescent wave, which preferentially samples material deposited directly onto the ATR crystal and is therefore less affected by the composition of the bulk solution. Since a relatively dense layer of AuNPs can be placed on the crystal surface, the interfacial-tobulk ratio can be maximized. Initially, a solution of $10 \mu \mathrm{mol} / \mathrm{L}$ BSA was introduced to an immobilized AuNP film at neutral $\mathrm{pH}$ to obtain surface saturation under $\mathrm{N}$-form conditions. Then, a 10 $\mu \mathrm{mol} / \mathrm{L}$ BSA solution preadjusted to different $\mathrm{pH}$ values was flushed through the ATR cell and allowed to equilibrate for $5 \mathrm{~min}$ before acquiring each spectrum. Figure $4 \mathrm{~b}$ shows the spectra of BSA conjugates on AuNPs as a function of $\mathrm{pH}$. Spectra are vertically adjusted for the purpose of clarity. The two adsorption bands are indicative of amide groups: amide I $\left(1600-1700 \mathrm{~cm}^{-1}\right)$ and amide II $\left(1500-1600 \mathrm{~cm}^{-1}\right) .^{71-73}$ The amide I region is mainly due to $\mathrm{C}=\mathrm{O}$ stretching, while the amide II region reflects in-plane $\mathrm{N}-\mathrm{H}$ bending and $\mathrm{C}-\mathrm{N}$ stretching. In general, amide II is less sensitive to protein conformation changes (secondary or tertiary structure) but is useful for quantification of protein adsorption in aqueous solution due to its reduced interference from the absorbance of molecular water $\left(\approx 1640 \mathrm{~cm}^{-1}\right)$ relative to amide I. Over a range of $\mathrm{pH}$ values from 2.2 to 7.1 , as shown in Figure $4 b$, the peak height of the IR absorbance for the amide II band is nearly constant (the peak height of absorbance is $0.07 \pm$ 0.006), indicating that molecular surface density is relatively unchanged in this $\mathrm{pH}$ range. This supports the supposition that the transition in $0.5 \Delta d_{\mathrm{p}, \mathrm{hz}}$ observed in Figure $4 \mathrm{a}$ is primarily due to a change in adsorbed BSA conformation. In section 3.6 we will continue to use IR to investigate the effect of $\mathrm{pH}$ on the secondary structure of BSA. In addition to $\mathrm{pH}$, we examined the influence of ionic strength on BSA structure over the range represented in the investigation reported here and found no observable dependence of $z$-average size (Supporting Information, Figure S3).

3.3. Characterization of BSA Conjugation on AuNPs in the Dry State. The number-weighted particle diameter, $d_{\mathrm{p}, \mathrm{m}}$ of conjugated and unconjugated AuNPs in the dry state (i.e., as aerosols) was obtained using ES-DMA. Figure $5 \mathrm{a}-\mathrm{c}$ summarizes the ES-DMA results and clearly illustrates a significant increase in $d_{\mathrm{p}, \mathrm{m}}$ (defined as $\Delta d_{\mathrm{p}, \mathrm{m}}$ ) following conjugation with BSA, even in the dry state. For nominally $10 \mathrm{~nm}$ AuNPs, the mean $d_{\mathrm{p}, \mathrm{m}}$ increases from 13.0 to $19.3 \mathrm{~nm}\left(\Delta d_{\mathrm{p}, \mathrm{m}}=6.3 \mathrm{~nm}\right)$. For $30 \mathrm{~nm}$ AuNPs, the mean $d_{\mathrm{p}, \mathrm{m}}$ increases from 31.1 to $37.2 \mathrm{~nm}\left(\Delta d_{\mathrm{p}, \mathrm{m}}=\right.$ $6.1 \mathrm{~nm})$, and for $60 \mathrm{~nm}$ AuNPs, the mean $d_{\mathrm{p}, \mathrm{m}}$ increased from 59.0 to $64.9 \mathrm{~nm}\left(\Delta d_{\mathrm{p}, \mathrm{m}}=5.9 \mathrm{~nm}\right)$. Thus, as the core size decreases, $\Delta d_{\mathrm{p}, \mathrm{m}}$ exhibits a slight increasing trend. The reason may be due to differences in BSA surface density as will be discussed in a later section. As shown in Table 1, the uncertainty in the mean diameter determined by ES-DMA measurements is significantly lower $(\approx 0.3 \mathrm{~nm})$ than $\Delta d_{\mathrm{p}, \mathrm{m}}$ resulting from $\mathrm{BSA}$ conjugation $(\approx 6 \mathrm{~nm})$, confirming that $\Delta d_{\mathrm{p}, \mathrm{m}}$ determined from the distributions in Figure 5 is statistically significant.

Because ES-DMA is a particle counting technique with high resolution, the number-weighted distribution generated by ES-DMA should be able to accurately reflect the actual distribution or spread in particle size. ${ }^{49,59,74,75}$ From the change in distribution width, we can draw some conclusions concerning the homogeneity of the molecular conjugation. Applying a coreshell model to the BSA-AuNP conjugates, ${ }^{48-50}$ the full width at half-maximum (fwhm) of the size distribution ${ }^{76}$ should increase only if conjugation is heterogeneous (i.e., varies across different particles) or if coated and uncoated particles coexist in the particle population following conjugation with BSA. Conversely, if a uniform coating is formed, the width of size distribution should be conserved following conjugation, though the peak itself should shift upward. ${ }^{4-50}$ As shown in Figure 5a-c, following conjugation with BSA, the fwhm were relatively unchanged for the three core sizes, indicating the formation of a homogeneous BSA shell over the AuNP core. In addition, the size distributions measured by ES-DMA show a single normal (Gaussian type) peak (i.e., no peak splitting or multimodal distribution), confirming the splitting shown in the AFFF data in Figure $3 \mathrm{a}$ is not attributable to heterogeneous (bimodal) BSA surface density or aggregation of AuNPs but is likely a measurement/fractionation artifact as previously suggested.

In comparing the increase in particle size due to BSA conjugation in different environments, we find that the increase in size measured in the dry state $\left(\Delta d_{\mathrm{p}, \mathrm{m}}\right)$ is about $6 \mathrm{~nm}$; this value is significantly lower than $\Delta d_{\mathrm{p}, \mathrm{hz}}$ measured in solution $(\approx 8-12$ $\mathrm{nm}$ by DLS and AFFF). This is consistent with our previous results for SH-PEG5K on AuNPs. ${ }^{50}$ In solution, the particle size measured by DLS or AFFF represents the hydrodynamic envelope of the particle-molecule conjugate presented as an equivalent sphere and is relatively less affected by the surface density in the monolayer regime. That is, a submonolayer of adsorbed protein, extending out from the surface and sweeping entrapped solution along with it as it diffuses, will greatly increase the hydrodynamic size relative to the native particle; further addition of protein toward a full monolayer will contribute minimally to the hydrodynamic envelope, as it tends to simply fill in gaps and does not further extend the envelope. On the other hand, $\Delta d_{\mathrm{p}, \mathrm{m}}$ measured in the dry state should be less than that measured in the wet state because solvent is removed and the protein is collapsed onto the surface during the ES-DMA analysis. Current results bear this out. In addition, it is found that BSA has a more compact conformation than SH-PEG5K; the ratio $\Delta d_{\mathrm{p}, \mathrm{m}} / \Delta d_{p, \mathrm{hz}}$ for BSA-AuNPs is much larger $(48-75 \%)$ relative to $\Delta d_{\mathrm{p}, \mathrm{m}} / \Delta d_{p, \mathrm{hz}}$ for SH-PEG5K-AuNPs $(\approx 25 \%) .{ }^{50}$

3.4. Quantification of Adsorbed Density. One advantage of ES-DMA is its capacity to quantify molecular surface density under certain conditions. ${ }^{48^{-}-50}$ To accurately convert $\Delta d_{\mathrm{p}, \mathrm{m}}$ obtained by ES-DMA to the number of molecules attached to the surface of AuNPs, it is important to have a constant molecular size during the measurements. However, as previously shown, BSA is known to undergo conformational changes with environmental variations (e.g., $\mathrm{pH}$ and ionic strength) while in the wet state. Moreover, conformational changes observed during solution phase measurements (i.e., by DLS) make it difficult to quantify surface density based on size increase alone. On the other hand, measuring the size change in the dry state avoids the convolution of surface density and conformation in the wet state, since interactions with the solution phase are removed during the aerosolizing process. Hence, the physical size of BSA can be assumed constant during the ES-DMA measurements, regardless of the solvent conditions used prior to analysis.

Since ES-DMA can be used to accurately measure the particle size of BSA-conjugated AuNPs with very high resolution and 

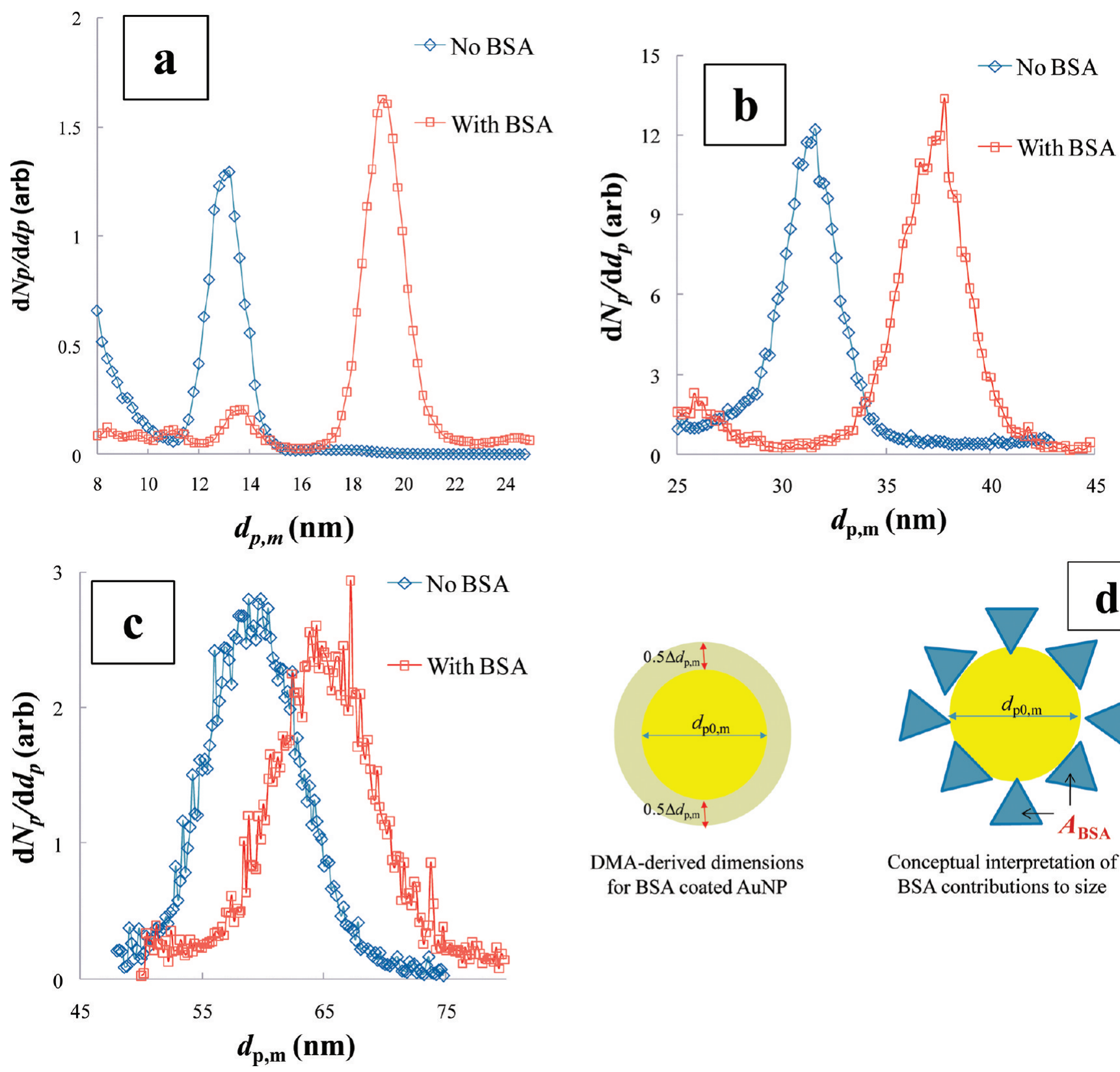

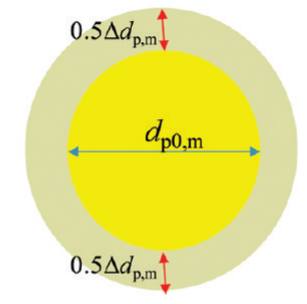

DMA-derived dimensions for BSA coated AuNP

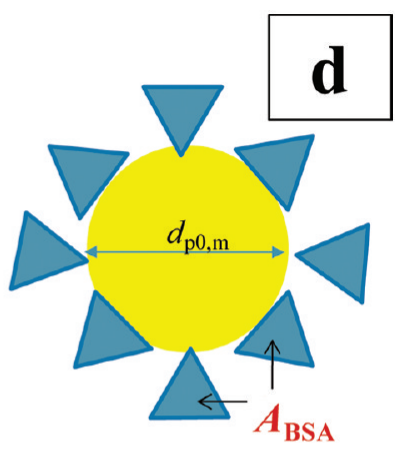

Conceptual interpretation of BSA contributions to size

Figure 5. Quantification of BSA conjugation on AuNPs by ES-DMA. (a) Particle size distributions of nominally $10 \mathrm{~nm}$ AuNPs. (b) Particle size distributions of nominally $30 \mathrm{~nm}$ AuNPs. (c) Particle size distributions of nominally $60 \mathrm{~nm}$ AuNPs. (d) Conceptual model for the calculation of molecular surface density via ES-DMA. $C_{\mathrm{BSA}}$ was $100 \mu \mathrm{mol} / \mathrm{L}$, and the experiment was conducted at $\mathrm{pH} 7$.

based on particle surface-to-charge ratio, the quantity of BSA molecules conjugated to AuNPs can be estimated from $\Delta d_{\mathrm{p}, \mathrm{m}} \cdot{ }^{48-50}$ A conceptual analytical model is shown in Figure $5 \mathrm{~d}$. From the increase in cross-sectional area of AuNPs, $\triangle A$, the molecular surface density of BSA on AuNPs, $\sigma$, can be obtained via ${ }^{48,49}$

$$
\Delta A=\pi\left[\left(d_{\mathrm{p} 0, \mathrm{~m}}+\Delta d_{\mathrm{p}, \mathrm{m}}\right)^{2}-d_{\mathrm{p} 0, \mathrm{~m}}^{2}\right] / 4=N A_{\mathrm{BSA}}
$$

Here, $d_{\mathrm{p} 0, \mathrm{~m}}$ is the diameter of core AuNPs measured by ES-DMA, and $A_{\mathrm{BSA}}$ is the cross-sectional area of BSA molecule. Using an equilateral triangular model for globular $\mathrm{N}$-form BSA with $8 \mathrm{~nm}$ edges and $3 \mathrm{~nm}$ thickness, ${ }^{31} A_{\mathrm{BSA}} \approx 25.9 \mathrm{~nm}^{2}$ (details of calculation of $A_{\mathrm{BSA}}$ are included in the Supporting Information). $N$ is the number of BSA molecules attached to the cross-sectional perimeter of the AuNPs $\left(\pi d_{\mathrm{p} 0, \mathrm{~m}}\right)$ and $N=N_{\mathrm{m}} \pi d_{p 0} . N_{\mathrm{m}}$ is the linear molecular density around the two-dimensional projection of the particles' circumference, ${ }^{48}$ which can be used to calculate $\sigma$ (where $\sigma=N_{\mathrm{m}}{ }^{2}$ ). Substituting $N$ in eq 2, $\sigma$ can be expressed as

$$
\sigma=\frac{\left[\left(d_{\mathrm{p} 0, \mathrm{~m}}+\Delta d_{\mathrm{p}, \mathrm{m}}\right)^{2}-d_{\mathrm{p} 0, \mathrm{~m}}^{2}\right]^{2}}{\left[4 d_{\mathrm{p} 0, \mathrm{~m}} A_{\mathrm{BSA}}\right]^{2}}
$$

Using $\Delta d_{\mathrm{p}, \mathrm{m}}$ and $d_{\mathrm{p} 0, \mathrm{~m}}$ measured in Figure $5 \mathrm{a}-\mathrm{c}$, the surface density of BSA, $\sigma$, is found to be $0.023 \pm 0.003 \mathrm{~nm}^{-2}$ for nominal $10 \mathrm{~nm}$ AuNPs, $0.017 \pm 0.002 \mathrm{~nm}^{-2}$ for nominal $30 \mathrm{~nm}$ AuNPs, and $0.014 \pm 0.002 \mathrm{~nm}^{-2}$ for nominal $60 \mathrm{~nm}$ AuNPs. These values correspond to 12,52 , and 153 molecules/particle for nominally 10, 30, and $60 \mathrm{~nm}$ AuNPs, respectively. Thus, the 
maximum surface density measured by ES-DMA ranges from $\approx 34 \%$ to $\approx 60 \%$ of the maximum monolayer surface density, $\approx 1 / A_{\mathrm{BSA}}\left(0.0386 \mathrm{~nm}^{-2}\right.$, assuming no spacing between BSA conjugates). The apparent submonolayer density could be attributed to intermolecular electrostatic repulsion and also steric hindrance between adsorbed BSA occupying neighboring sites on the $\mathrm{Au}$ surface. Modeling this "jamming effect" (i.e., space-limiting due to the mutual repulsion between adsorbed BSA molecules) has been shown to decrease the surface density as much as $71 \%$ of the maximum monolayer density depending on the molecular dimension used for the model. ${ }^{77,78}$ Note that the core-shell model (eq 3) is applicable for the calculation of $\sigma$ when the number of adsorbate molecules attached to the AuNP is extensively higher than unity. If the core size is less than about $10 \mathrm{~nm}$, a heterogeneous aggregation model may be more appropriate, but this is beyond the scope of the present work.

Fluorescence spectrometry was used for semiquantitative comparison and confirmation of the ES-DMA results. An advantage of using fluorescence is that surface density can be determined using the same suspension measured by ES-DMA. Because more than $99.98 \%$ of unbound BSA was removed from the AuNP samples, the fluorescence signals, and the corresponding BSA concentration, can be mostly attributed to the BSA bound to AuNPs. Knowing the total surface of AuNPs, $\sigma$ based on fluorescence can be obtained with the corresponding fluorescence signals.

As measured, $\sigma$ based on fluorescence is determined to be $0.010 \pm 0.005 \mathrm{~nm}^{-2}$ for nominal $30 \mathrm{~nm}$ AuNPs and $0.008 \pm$ $0.002 \mathrm{~nm}^{-2}$ for nominal $60 \mathrm{~nm}$ AuNPs. Because of poor recovery of AuNPs following the required centrifugation cleaning process, surface density for nominal $10 \mathrm{~nm}$ AuNPs appears unrealistically low $\left(0.005 \pm 0.001 \mathrm{~nm}^{-2}\right)$ and is therefore considered unreliable. Similar recovery issues were observed in the processing of samples for ES-DMA, but in this case results are not dependent on recovery. Nevertheless, in general, the surface density measured by ES-DMA is of the same order of magnitude as the results by fluorescence spectrometry. In addition, the results are consistent with reported values for BSA on Au surfaces, ranging from 0.015 to $0.03 \mathrm{~nm}^{-2}$. $6,27,63$

The ES-DMA and fluorescence results suggest a dependence of surface density (or adsorption) on particle (core) size. Several research groups have examined the effect of core size of AuNPs on surface density for large molecules using visible extinction spectroscopy and fluorescence spectrometry. $27,79,80$ Their results indicate that smaller size particles may permit a higher molecular packing density. This dependency is especially true when the core size is less than about $30 \mathrm{~nm}$ in diameter, qualitatively consistent with our observation by ES-DMA. One possible explanation for this size dependency is the geometric curvature effect, which dictates that smaller particles (higher arc) can allow a higher packing density of macromolecules, such as BSA. Additionally, surface faceting, a characteristic associated with citrate stabilized AuNPs, may have a significant effect. Although the exact role is unconfirmed at this time, we speculate that the size and number of facets change with the core size and, if this is true, would be expected to have a measurable geometric effect on adsorbate density. For instance, nominally 60 $\mathrm{nm}$ AuNPs appear to have larger and fewer facets per particle relative to $10 \mathrm{~nm}$ AuNPs, based on electron microscopy images. ${ }^{75}$ Table 2 summarizes the maximum surface density of BSA on AuNPs.
Table 2. Summary of Maximum Surface Density of BSA on AuNPs $\left(\sigma_{\max }\right)$ Estimated from Results Obtained by ES-DMA and Fluorescence Spectrometry

\begin{tabular}{ccc} 
nominal size $(\mathrm{nm})$ & $\sigma_{\max }$ ES-DMA $\left(\mathrm{nm}^{-2}\right)$ & $\sigma_{\max }$ fluorescence $\left(\mathrm{nm}^{-2}\right)$ \\
10 & $0.023 \pm 0.003$ & $0.005 \pm 0.001$ \\
30 & $0.017 \pm 0.002$ & $0.010 \pm 0.005$ \\
60 & $0.014 \pm 0.002$ & $0.008 \pm 0.002$ \\
\hline
\end{tabular}

3.5. Adsorption Isotherms. In the previous sections, we probed the formation of BSA-AuNP conjugates and studied BSA surface density and conformation using complementary and orthogonal characterization approaches. Next, we employ and compare different methods to obtain the adsorption isotherm for BSA on AuNPs. By characterizing $\sigma$ as a function of $C_{\mathrm{BSA}}$ at constant temperature, we can quantify the binding affinity for BSA on AuNPs by obtaining an equilibrium binding constant, $K$, which can be used, for example, as an important indicator in formulation design for nanomedicine applications.

Figure 6a shows the change in particle size measured as a function of $C_{\mathrm{BSA}}$. Nominal $60 \mathrm{~nm}$ AuNPs are employed in this study. We normalize the change of hydrodynamic diameter $\left(\Delta d_{\mathrm{p}, \mathrm{hz}}\right)$ and the change of aerodynamic diameter $\left(\Delta d_{\mathrm{p}, \mathrm{m}}\right)$ relative to their maximum values at the highest $C_{\mathrm{BSA}}\left(\Delta d_{\mathrm{p}, \mathrm{hz}, \max }\right.$ and $\Delta d_{\mathrm{p}, \mathrm{m}, \max }$, respectively); in other words, $\Delta d_{\mathrm{p}, \mathrm{hz}}{ }^{*}=\Delta d_{\mathrm{p}, \mathrm{hz}} / \Delta d_{\mathrm{p}, \mathrm{hz}, \max }$ and $\Delta d_{\mathrm{p}, \mathrm{m}}{ }^{*}=\Delta d_{\mathrm{p}, \mathrm{m}} / \Delta d_{\mathrm{p}, \mathrm{m}, \max }$. As shown in Figure 6a, increasing $C_{\mathrm{BSA}}$ increases $\Delta d_{\mathrm{p}, \mathrm{m}}{ }^{*}$ and $\Delta d_{\mathrm{p}, \mathrm{hz}}{ }^{*}$ at lower $C_{\mathrm{BSA}}(<10 \mu \mathrm{mol} / \mathrm{L})$, showing an increase in the amount of adsorbed BSA on the surface of the AuNPs. At $C_{\mathrm{BSA}}>10 \mu \mathrm{mol} / \mathrm{L}, \Delta d_{\mathrm{p}} / \Delta d_{\mathrm{p}, \max }$ plateaus, indicating $\sigma$ has attained a saturation level under these conditions. In our previous study, ${ }^{50}$ DLS was found to provide better sensitivity than ES-DMA for probing the initial formation of SH-PEG5K-AuNP conjugates. In the present case, DLS and ES-DMA provide statistically identical results for $\Delta d_{\mathrm{p}} / \Delta d_{\mathrm{p} \text {, max }}$ versus $C_{\mathrm{BSA}}$, indicating that these techniques have the same sensitivity to probe the conjugation of BSA and AuNPs.

Using eq $3, \Delta d_{\mathrm{p}, \mathrm{m}}{ }^{*}$ is converted to $\sigma / \sigma_{\max }$ where $\sigma_{\max }$ is the maximum surface density of BSA calculated from the measurement results (plateau value). As shown in Figure $6 \mathrm{~b}$ (diamond symbols), $\sigma / \sigma_{\max }$ reaches $90 \%$ when $C_{\mathrm{BSA}} \approx 30 \mu \mathrm{mol} / \mathrm{L}$. Using the Langmuir adsorption model to obtain the equilibrium binding constant, $K$

$$
K=\frac{1}{C_{\mathrm{BSA}}\left[\left(\sigma_{\max } / \sigma\right)-1\right]}
$$

As shown in Figure 6b (square symbols and line fit), a linear relationship between $1 /\left[\sigma_{\max } / \sigma-1\right]$ and $C_{\mathrm{BSA}}$ exists with a slope, $K=0.51 \times 10^{6} \mathrm{~L} / \mathrm{mol}$. This represents a relatively high binding constant $\left(>10^{4} \mathrm{~L} / \mathrm{mol}\right)$ indicating a strong affinity for BSA on the AuNP surface, as would be expected if thiol-mediated bonding is involved (versus a purely physical adsorption mechanism). This value is consistent with previously reported values in the literature $\left(\approx 1 \times 10^{6} \mathrm{~L} / \mathrm{mol}^{6,9}\right)$.

Figure $7 \mathrm{a}$ shows the adsorption isotherm measured by fluorescence assay using the same suspensions in Figure 6. Again, $\sigma / \sigma_{\max }$ reaches $\approx 90 \%$ when $C_{\mathrm{BSA}} \approx 30 \mu \mathrm{mol} / \mathrm{L}$, consistent with the ES-DMA results above. Using eq 4 , the equilibrium binding constant is estimated to be $0.62 \times 10^{6} \mathrm{~L} / \mathrm{mol}$. Figure $7 \mathrm{~b}$ shows an adsorption isotherm obtained using a method based on ATRFTIR. To quantify the relative surface density of BSA on AuNPs 

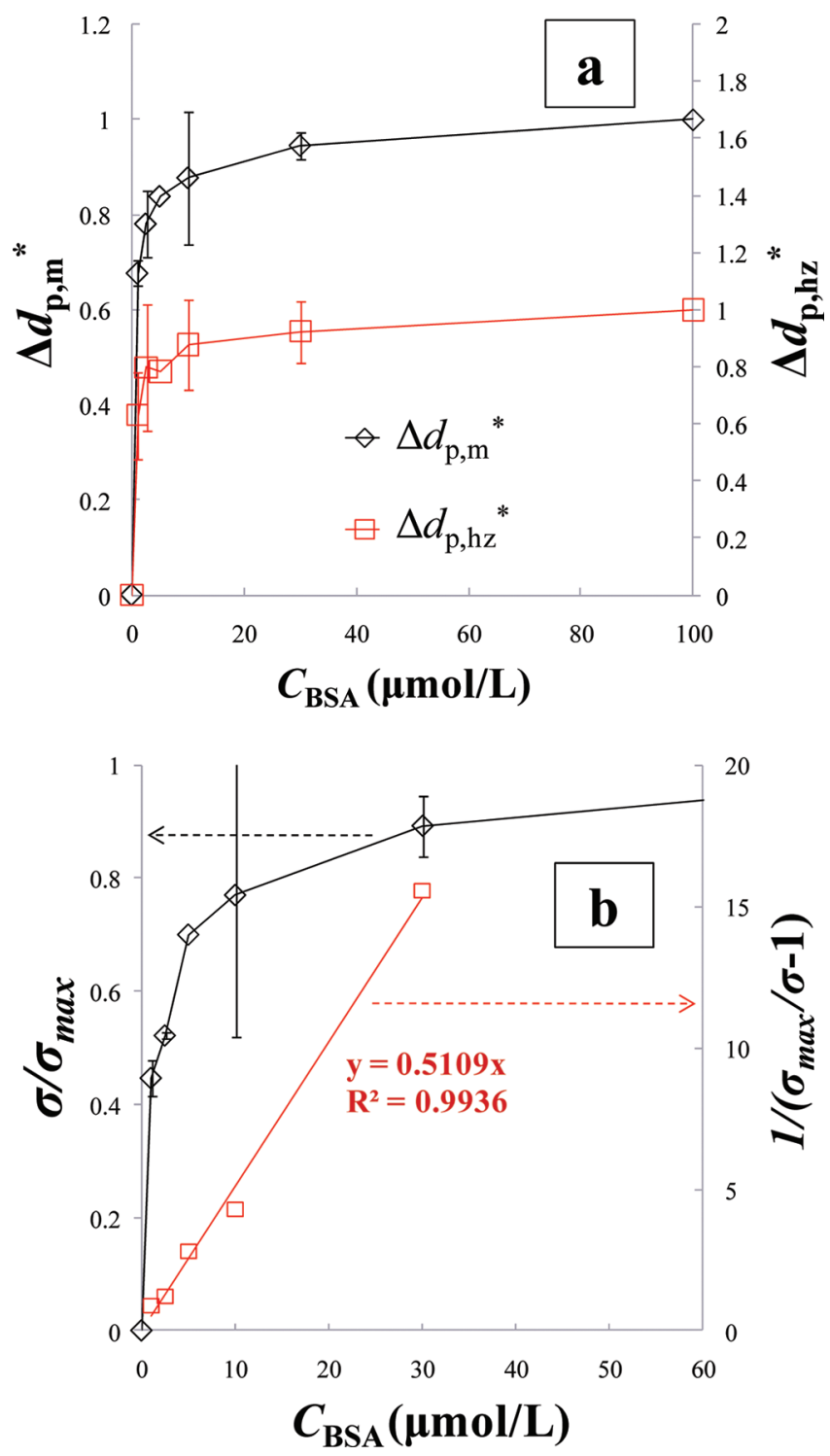

Figure 6. Adsorption isotherms for BSA on nominal $60 \mathrm{~nm}$ AuNPs obtained using physical measurement methods. Experimental temperature is $21^{\circ} \mathrm{C}$. (a) Dependence of the measured increase in particle size on $C_{\mathrm{BSA}}: \Delta d_{\mathrm{p}, \mathrm{m}}{ }^{*}$ (diamonds) and $\Delta d_{\mathrm{p}, \mathrm{hz}}{ }^{*}$ : (squares). (b) Calculated adsorption isotherm (diamonds and squares, based on ES-DMA) presented as fractional surface density; line is a guide to the eyes. Fit of data (line) to Langmuir adsorption equation. The slope $K$ of the Langmuir fit gives the equilibrium binding constant. Error bars are one standard deviation based on two replicates measured under repeatability conditions. The $\mathrm{pH}$ during isotherm experiments was between 7.3 and 7.7.

by ATR-FTIR, we determine the peak heights at $1540 \mathrm{~cm}^{-1}$ (amide II) as a function of $C_{\mathrm{BSA}}$ in the flow cell and then normalize the peak heights by the maximum peak intensity to obtain $\sigma / \sigma_{\max }$. This approach assumes that peak height scales proportionately with the adsorbed quantity of BSA; the results shown in Figure $7 \mathrm{~b}$ indicate that $\sigma / \sigma_{\max }$ reaches $\approx 90 \%$ at a concentration of about $10 \mu \mathrm{mol} / \mathrm{L}$. Using eq 4 , an equilibrium binding constant of $1.65 \times 10^{6} \mathrm{~L} / \mathrm{mol}$ is obtained, slightly higher than the values obtained by ES-DMA and fluorescence, but of similar magnitude (Figures $6 \mathrm{~b}$ and $7 \mathrm{a}$ ).
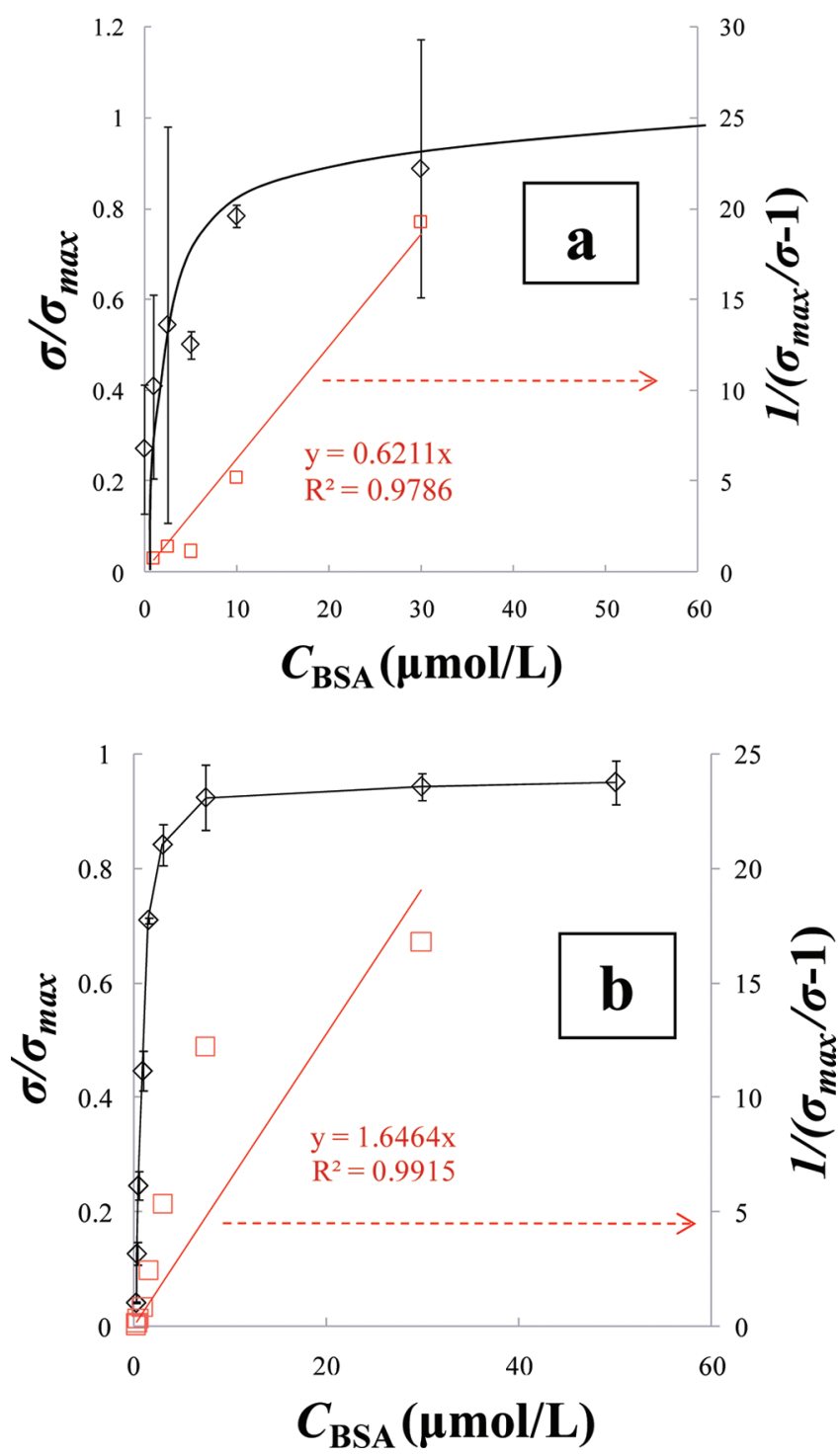

Figure 7. Adsorption isotherms (diamond symbols) for BSA on nominal $60 \mathrm{~nm}$ AuNPs obtained by spectroscopic methods. Experimental temperature is $21^{\circ} \mathrm{C}$. (a) Fluorescence spectrometry. (b) ATRFTIR. Lines are a guide to the eyes. Data (square symbols) and fit to Langmuir model. Error bars represent one standard deviation for at least two replicate measurements. The slope $K$ of the Langmuir fit is the equilibrium binding constant. The $\mathrm{pH}$ during isotherm measurements was between 7.0 and 7.4, but the initial native AuNP was unbuffered with a $\mathrm{pH}$ at 6.0 .

3.6. Effect of Adsorption and pH on BSA Secondary Structure. ATR-FTIR can be applied to investigate changes in the secondary structure of BSA after it is conjugated to the AuNP surface. Previously, IR spectroscopy has been used to investigate the interaction of BSA on flat gold surfaces, ${ }^{71-73}$ using the amide I region to isolate changes in protein conformation. Differences in the amide bond orientation and the protein backbone are due to a varying secondary structure giving rise to various vibrational frequencies: ${ }^{72} \alpha$-helix $\left(1650-1655 \mathrm{~cm}^{-1}\right), \beta$-sheet or $\beta$-turn $\left(1663-1685 \mathrm{~cm}^{-1}\right)$, random chains $\left(1644-1648 \mathrm{~cm}^{-1}\right)$, extended chains $\left(1635-1639 \mathrm{~cm}^{-1}\right)$, and extended chains plus $\beta$-sheet $\left(1621-1632 \mathrm{~cm}^{-1}\right)$. Therefore, the overall shape and 

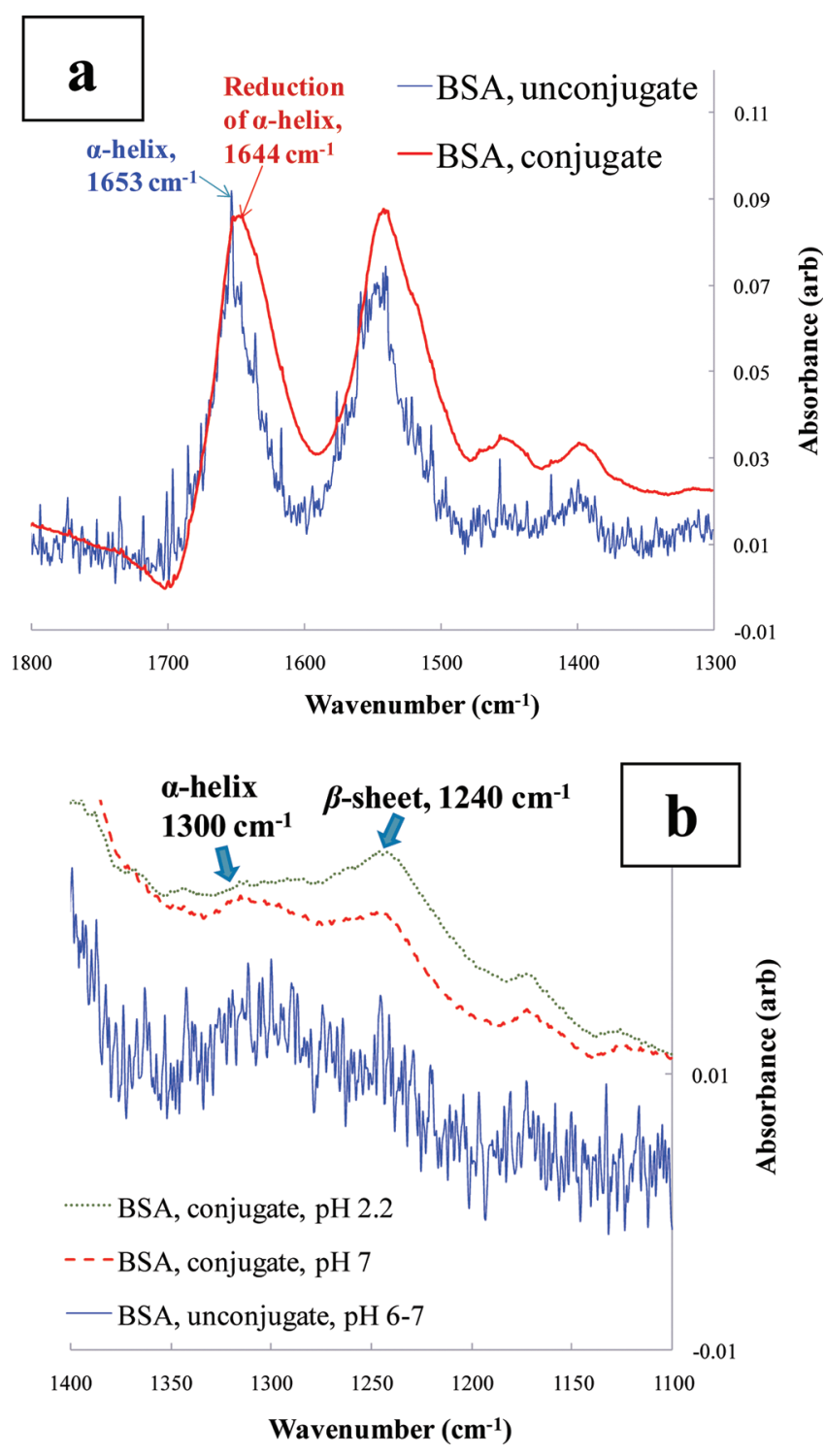

Figure 8. Comparison of ATR-FTIR spectra of unconjugated (free) BSA and BSA-AuNP conjugate: (a) amide I and amide II regions, $\mathrm{pH}$ between 6 and 7; (b) amide III region. The IR absorbance of unconjugated BSA is multiplied $30 \times$ for the purpose of comparison.

maxima of this amide I band are determined by the secondary structure of the protein. By deconvoluting the peak of the amide I region, Roach et al. studied changes in secondary structure of BSA following binding to different types of flat surfaces. ${ }^{72}$ The authors determined that BSA is denatured by the interaction with an alkanethiol-functionalized Au film, a hydrophobic surface, in the process losing a large degree of their $\alpha$-helix structure.

Presently we apply this approach to the analysis of BSA on spheroidal (but faceted) nominal $60 \mathrm{~nm}$ AuNPs. As shown in Figure $8 \mathrm{a}$, the amide I peak for unconjugated BSA is centered at $1653 \mathrm{~cm}^{-1}$, indicating that the principal secondary structure of free BSA at $\mathrm{pH} 7$ is the $\alpha$-helix form. After conjugation to AuNPs, the center of the amide I peak shifts down to $1644 \mathrm{~cm}^{-1}$. The lower frequency regions represent less compact structures, as described above. Hence, the reduction of frequency indicates a change from the $\alpha$-helix to more random and open chains. In addition to the peak frequency, the amide I band broadens asymmetrically to the right of the center frequency following conjugation, which indicates an increase in $\beta$-sheet content, random chains, or extended chains. These results, taken together, suggest that the secondary structure of BSA is changed in the process of conjugating with AuNPs.

Additional evidence for conformation change can be obtained by investigating the amide III region between 1200 and 1350 $\mathrm{cm}^{-1}$. For BSA, the peak frequency at $1300 \mathrm{~cm}^{-1}$ is attributed to $\alpha$-helix, and the peak frequency from 1235 to $1260 \mathrm{~cm}^{-1}$ corresponds to $\beta$-sheet. ${ }^{81}$ Kaiden et al. investigated the denaturing of unbound BSA in solution, and they found not only that the frequency of amide I region was shifted to a lower value after denaturing but also that the peak intensities in the amide III region were altered. In our experiments, as shown in Figure 8b, after conjugation to AuNPs at neutral $\mathrm{pH}$ (red), the peak intensity at $1300 \mathrm{~cm}^{-1}$ was significantly decreased accompanied by the increase of the peak intensity at $1240 \mathrm{~cm}^{-1}$, confirming the conformation of BSA conjugates are changed and transformed from $\alpha$-helix to be $\beta$-sheet at the surface of the AuNP.

Looking into the effect of $\mathrm{pH}$ on the conformation of BSA conjugates, we saw a significant change in the amide III region by lowing $\mathrm{pH}$ from 7 (red, dashed) to 2.2 (green, dotted), even though the difference in the amide I region was less significant (Figure $4 \mathrm{~b}$ and Figure S4 in Supporting Information). This result indicates possibly less $\alpha$-helix presenting in the BSA conjugates at acidic $\mathrm{pH}$ relative to neutral $\mathrm{pH}$, and a possible explanation may be the reduction of intermolecular electrostatic interaction, similar to the free BSA in different $\mathrm{pH}$ environments. $^{12,28,31,34,36,46,69}$

Secondary structures of proteins are important to proteins functionality, in particular its biological activity. For instance, altering the secondary structure of proteins can significantly impact the corresponding enzyme activity. ${ }^{82,83}$ Once proteins are bound to nanoparticles, the change of the secondary structure induced by the surface interaction may impact the corresponding enzyme activity of the protein-NP conjugate. For example, Wang et al. found a $36 \%$ decrease of enzyme activity when trypsin was immobilized on $\mathrm{TiO}_{2}$, with a significant corresponding change in the secondary structure. ${ }^{83}$ In the present work we use the BSA-AuNP conjugate as a model system and employ ATR-FTIR to investigate the change of BSA secondary structure following conjugate formation. The method proposed here is not limited to BSA-AuNPs and can be used to investigate other types of protein-NP systems. By characterizing the change of protein secondary structures under various conditions, one should be able to correlate this to the corresponding enzyme activity using in vitro and in vivo methods.

\section{CONCLUSIONS}

We have demonstrated that conjugation of BSA with AuNPs can be characterized quantitatively and qualitatively by applying complementary, and sometimes orthogonal, physical and spectroscopic methods. In the present study we use DLS, AFFF, ESDMA, fluorescence spectrometry, and ATR-FTIR to obtain information regarding both molecular surface density and molecular conformation for BSA adsorbed onto AuNPs. For larger diameter AuNPs (nominally $60 \mathrm{~nm}$ ) with a relatively lower contribution of scattered light arising from unbound BSA (i.e., a high $\gamma$ ratio as defined previously), DLS exhibits reasonably good accuracy for probing the formation of BSA-AuNP 
conjugates, and the results are consistent with those obtained by fractionation and high-resolution sizing via AFFF. For nominally smaller AuNP cores, interference from free BSA can skew results and complicate their interpretation unless additional steps are taken to remove the interference. The in situ physical characterization in the wet state (e.g., using DLS) explores the capacity to monitor the change of molecular conformation of BSA (or other globular proteins) on AuNPs under physiologically relevant conditions and in real time.

At similar BSA surface densities, we find a larger increase of BSA-AuNP size at $\mathrm{pH}<2.5$ than at a more basic $\mathrm{pH}$ values $(\mathrm{pH}$ 3.4-7.3), indicating a more expanded conformation under strongly acidic conditions corresponding to the so-called $\mathrm{E}$ or $\mathrm{F}$ forms of BSA. Moreover, we are able to exclude agglomeration as a source of particle size increase. When modifying $\mathrm{pH}$ between 3.4 and 7.3, the particle size measured by DLS is essentially constant, indicating the conformation of BSA conjugates is relatively stable over this $\mathrm{pH}$ range, and presumably represents the $\mathrm{N}$ form of BSA. This result is contrasted with free BSA, which is known to undergo at least two structural transformations over this $\mathrm{pH}$ range. ES-DMA has been used to characterize the particle size in the dry (aerosolized) state, where BSA surface density can be calculated from the measured change of aerosol size. The maximum surface density for BSA on AuNPs measured by ES-DMA and fluorescence spectrometry is dependent on the AuNP core size and consistent across characterization techniques. Adsorption isotherms obtained by multiple methods, including DLS, ES-DMA, fluorescence spectrometry, and ATRFTIR, exhibit consistency across methods, with BSA surface density approaching $90 \%$ when $C_{\mathrm{BSA}}$ is $\approx 10-30 \mu \mathrm{mol} / \mathrm{L}$. Using the Langmuir adsorption model to quantify the binding affinity, we obtain a surface binding constant for BSA on AuNPs $\approx 0.51 \times 10^{6} \mathrm{~L} / \mathrm{mol}$ by ES-DMA, $\approx 0.62 \times 10^{6} \mathrm{~L} / \mathrm{mol}$ by fluorescence assay, and $1.65 \times 10^{6} \mathrm{~L} / \mathrm{mol}$ by ATR-FTIR. Thiolgold binding at the interface may induce or modify the observed conformation change of BSA, and the secondary structure of BSA appears to be modified upon adsorption, resulting in more open structures and less $\alpha$-helix structure. Factors that could potentially limit the applicability of this approach include the necessity to have robust binding between the adsorbates and the NP surface (in order to avoid desorption during the cleaning process required to remove excess adsorbate from the solution phase prior to fluorescence and ES-DMA measurements) and NP dimensions that approach the molecular size of the adsorbate (making it more difficult to separate the free and bound species and complicating the analysis of isotherms and size measurements).

Besides the adsorption of albumin on AuNPs, the proposed complementary characterization approach can also be used to study adsorption/desorption processes of other globular proteins onto/from various types of nanoparticle systems. Through the quantitative study of particle-protein interactions, as demonstrated in this paper, parameters such as equilibrium binding constants and adsorption/desorption rate constants can be obtained and then used to improve the formulation design and quality assurance of nanomedicine products. The work presented here provides proof of concept and demonstrates the efficacy of the complementary characterization approach for the investigation of protein conjugation in nanoparticle systems. This work also serves as a template for the application of commonly used methods, such as DLS and fluorescence spectrometry, for investigating the interaction of nanoparticles with proteins and other surface-active molecular species.

\section{ASSOCIATED CONTENT}

S Supporting Information. Additional information on characterizing BSA-conjugated AuNP. This material is available free of charge via the Internet at http://pubs.acs.org.

\section{AUTHOR INFORMATION}

\section{Corresponding Author}

*E-mail: vince.hackley@nist.gov.

\section{ACKNOWLEDGMENT}

The authors acknowledge Dr. Anil Patri of the National Cancer Institute's Nanotechnology Characterization Laboratory for helpful discussions in advance of the present work. The authors thank Dr. Richard Gates at the National Institute of Standards and Technology (NIST) for his assistance with the ATR-FTIR measurements and Ms. Melissa Davila-Morris, a Summer Undergraduate Research Fellow at NIST, for her assistance with sample preparation for the fluorescence and ATR-FTIR measurements. In addition, the authors also thank Dr. Julian Taurozzi, Dr. Robert Cook, and Suvajyoti Guha at NIST for the review and suggestions to the manuscript.

\section{REFERENCES}

(1) The findings and conclusions in this article have not been formally disseminated by the Food and Drug Administration and should not be construed to represent any Agency determination or policy.

(2) Paciotti, G.; Li, Q.; Simpson, B.; Soltesiak, K.; Meyer, L.; Davis, N.; Tamarkin, L. Eur. Cytokine Network 1998, 9 (3), 536.

(3) Paciotti, G. F.; Myer, L. D.; Kim, T. H.; Wang, S.; Alexander, H. R.; Weinreich, D.; Tamarkin, L. Clin. Cancer Res. 2001, 7 (11), 3673S-3674S.

(4) Paciotti, G. F.; Myer, L.; Weinreich, D.; Goia, D.; Pavel, N.; McLaughlin, R. E.; Tamarkin, L. Drug Delivery 2004, 11 (3), 169-183.

(5) Paciotti, G. F.; Kingston, D. G. I.; Tamarkin, L. Drug Dev. Res. 2006, 67 (1), 47-54.

(6) Brewer, S. H.; Glomm, W. R.; Johnson, M. C.; Knag, M. K.; Franzen, S. Langmuir 2005, 21 (20), 9303-9307.

(7) Wangoo, N.; Suri, C. R.; Shekhawat, G. Appl. Phys. Lett. 2008.

(8) Eck, W.; Craig, G.; Sigdel, A.; Ritter, G.; Old, L. J.; Tang, L.; Brennan, M. F.; Allen, P. J.; Mason, M. D. ACS Nano 2008, 2 (11), 2263-2272.

(9) Lacerda, S. H. D.; Park, J. J.; Meuse, C.; Pristinski, D.; Becker, M. L.; Karim, A.; Douglas, J. F. ACS Nano 2010, 4 (1), 365-379.

(10) Lynch, I.; Dawson, K. A. Nano Today 2008, 3 (1-2), 40-47.

(11) Dobrovolskaia, M. A.; Patri, A. K.; Zheng, J. W.; Clogston, J. D.; Ayub, N.; Aggarwal, P.; Neun, B. W.; Hall, J. B.; Mcneil, S. E. Nanomed.Nanotechnol. Biol. Med. 2009, 5 (2), 106-117.

(12) Carter, D. C.; Ho, J. X. Adv. Protein Chem. 1994, 45 (45), 153-203.

(13) Ho, J. X.; Holowachuk, E. W.; Norton, E. J.; Twigg, P. D.; Carter, D. C. Eur. J. Biochem. 1993, 215 (1), 205-212.

(14) Goodman, D. S. J. Am. Chem. Soc. 1958, 80 (15), 3892-3898.

(15) Ao, L. M.; Gao, F.; Pan, B. F.; Cui, D. X.; Gu, H. C. Chin. J. Chem. 2006, 24 (2), 253-256.

(16) Chadborn, N.; Bryant, J.; Bain, A. J.; O’Shea, P. Biophys. J. 1999, 76 (4), 2198-2207.

(17) Moulin, A. M.; O'Shea, S. J.; Badley, R. A.; Doyle, P.; Welland, M. E. Langmuir 1999, 15 (26), 8776-8779.

(18) Figge, J.; Rossing, T. H.; Fencl, V. J. Lab. Clin. Med. 1991, 117 (6), 453-467. 
(19) Porter, D.; Sriram, K.; Wolfarth, M.; Jefferson, A.; SchweglerBerry, D.; Andrew, M.; Castranova, V. Nanotoxicology 2008, 2 (3), $144-154$.

(20) Sager, T. M.; Porter, D. W.; Robinson, V. A.; Lindsley, W. G.; Schwegler-Berry, D. E.; Castranova, V. Nanotoxicology 2007, 1 (2), 118-129.

(21) Bihari, P.; Vippola, M.; Schultes, S.; Praetner, M.; Khandoga, A. G.; Reichel, C. A.; Coester, C.; Tuomi, T.; Rehberg, M.; Krombach, F. Part. Fibre Toxicol. 2008.

(22) Ji, Z.; Jin, X.; George, S.; Xia, T.; Meng, H.; Wang, X.; Suarez, E.; Zhang, H.; Hoek, E. M. V.; Godwin, H.; Nel, A. E.; Zink, J. I. Environ. Sci. Technol. 2010, DOI 10.1021/es100417s.

(23) Schulze, C.; Kroll, A.; Lehr, C. M.; Schafer, U. F.; Becker, K.; Schnekenburger, J.; Isfort, C. S.; Landsiedel, R.; Wohlleben, W. Nanotoxicology 2008, 2 (2), 51-U17.

(24) Tantra, R.; Tompkins, J.; Quincey, P. Colloids Surf., B 2010, 75 (1), 275-281.

(25) Song, L. Sorption of Bovine Serum Albumin on Nano and Bulk Oxide Particles. MS Dissertation, University of MassachusettsAmherst, 2009.

(26) Estey, T.; Kang, J.; Schwendeman, S. P.; Carpenter, J. F. J. Pharm. Sci. 2006, 95 (7), 1626-1639.

(27) Teichroeb, J. H.; Forrest, J. A.; Jones, L. W. Eur. Phys. J. E: Soft Matter Biol. Phys. 2008, 26 (4), 411-415.

(28) Peters, T. Adv. Protein Chem. 1985, 37, 161-245.

(29) Era, S.; Sogami, M. J. Pept. Res. 1998, 52 (6), 431-442.

(30) Hirayama, K.; Akashi, S.; Furuya, M.; Fukuhara, K. Biochem. Biophys. Res. Commun. 1990, 173 (2), 639-646.

(31) Lee, C. T.; Smith, K. A.; Hatton, T. A. Biochemistry 2005, 44 (2), 524-536.

(32) Brown, J. R. Fed. Proc. 1975, 34 (3), 591.

(33) Cannistraro, S.; Sacchetti, F. Phys. Rev. A 1986, 33 (1), 745-746.

(34) Khan, M. Y. Biochem. J. 1986, 236 (1), 307-310.

(35) Putnam, F. W. The Plasma Proteins, 2nd ed.; Academic Press: New York, 1975; Vol. 1.

(36) Harrington, W. F.; Johnson, P.; Ottewill, R. H. Biochem. J. 1956, 62 (4), 569-582.

(37) Katchalski, E.; Benjamin, G. S.; Gross, V. J. Am. Chem. Soc. 1957, 79 (15), 4096-4099.

(38) Cedervall, T.; Lynch, I.; Lindman, S.; Berggard, T.; Thulin, E.; Nilsson, H.; Dawson, K. A.; Linse, S. Proc. Natl. Acad. Sci. U.S.A. 2007, 104 (7), 2050-2055.

(39) Fujiwara, K.; Watarai, H.; Itoh, H.; Nakahama, E.; Ogawa, N. Anal. Bioanal. Chem. 2006, 386 (3), 639-644.

(40) Housni, A.; Ahmed, M.; Liu, S. Y.; Narain, R. J. Phys. Chem. C 2008, 112 (32), 12282-12290.

(41) Krasovskii, V. I.; Nagovitsyn, I. A.; Chudinova, G. K.; Savranskii, V. V.; Karavanskii, V. A. Bull. Lebedev Phys. Inst. 2007, 34 (11), 321-324.

(42) Pan, B. F.; Cui, D. X.; Xu, P.; Li, Q.; Huang, T.; He, R.; Gao, F. Colloids Surf., A 2007, 295 (1-3), 217-222.

(43) Reilly, J. P. O.; Fisk, J. D.; Rooth, M.; Perkins, E.; Shaw, A. M. Phys. Chem. Chem. Phys. 2007, 9 (3), 344-345.

(44) Nakata, S.; Kido, N.; Hayashi, M.; Hara, M.; Sasabe, H.; Sugawara, T.; Matsuda, T. Biophys. Chem. 1996, 62 (1-3), 63-72.

(45) Silin, V.; Weetall, H.; Vanderah, D. J. J. Colloid Interface Sci. 1997, 185 (1), 94-103.

(46) Shang, L.; Wang, Y. Z.; Jiang, J. G.; Dong, S. J. Langmuir 2007, 23 (5), 2714-2721.

(47) MacCuspie, R. I.; Allen, A. J.; Hackley, V. A. Nanotoxicology 2010, DOI 10.3109/17435390.2010.504311.

(48) Pease, L. F.; Tsai, D. H.; Zangmeister, R. A.; Zachariah, M. R.; Tarlov, M. J. J. Phys. Chem. C 2007, 111 (46), 17155-17157.

(49) Tsai, D. H.; Zangmeister, R. A.; Pease, L. F.; Tarlov, M. J.; Zachariah, M. R. Langmuir 2008, 24 (16), 8483-8490.

(50) Tsai, D. H.; Delrio, F. W.; MacCuspie, R. I.; Cho, T. J.; Zachariah, M. R.; Hackley, V. A. Langmuir 2010, 26 (12), 1032510333.
(51) Pease, L. F.; Lipin, D. I.; Tsai, D. H.; Zachariah, M. R.; Lua, L. H. L.; Tarlov, M. J.; Middelberg, A. P. J. Biotechnol. Bioeng. 2009, 102 (3), 845-855.

(52) Corrigan, T. D.; Guo, S.; Phaneuf, R. J.; Szmacinski, H. J. Fluoresc. 2005, 15 (5), 777-784.

(53) Guo, S. H.; Tsai, S. J.; Kan, H. C.; Tsai, D. H.; Zachariah, M. R.; Phaneuf, R. J. Adv. Mater. 2008, 20 (8), 1424.

(54) Tsai, D. H.; Hawa, T.; Kan, H. C.; Phaneuf, R. J.; Zachariah, M. R. Nanotechnology 2007.

(55) Hackley, V. A.; Clogston, J. D. NIST-NCL Joint Assay Protocol PCC-1.

(56) Certain commercial equipment, instruments, or materials are identified in this report in order to specify the experimental procedure adequately. Such identification is not intended to imply recommendation or endorsement by the National Institute of Standards and Technology or the Department of Health and Human Services nor is it intended to imply that the materials or equipment identified are necessarily the best available for the purpose.

(57) Pease, L. F., III; Tsai, D. H.; Hertz, J. L.; Zangmeister, R. A.; Zachariah, M. R.; Tarlov, M. J. Langmuir 2010, 26 (13), 11384-11390.

(58) Pease, L. F.; Elliott, J. T.; Tsai, D. H.; Zachariah, M. R.; Tarlov, M. J. Biotechnol. Bioeng. 2008, 101 (6), 1214-1222.

(59) Tsai, D. H.; Pease, L. F.; Zangmeister, R. A.; Tarlov, M. J.; Zachariah, M. R. Langmuir 2009, 25 (1), 140-146.

(60) Cho, T. J.; Hackley, V. A. Anal. Bioanal. Chem. 201010.1007/ s00216-010-4133-6.

(61) ISO 13321:1996(E). Particle size analysis - Photon correlation spectroscopy, 1996.

(62) ISO 22412:2008, 2008.

(63) Kaufman, E. D.; Belyea, J.; Johnson, M. C.; Nicholson, Z. M.; Ricks, J. L.; Shah, P. K.; Bayless, M.; Pettersson, T.; Feldoto, Z.; Blomberg, E.; Claesson, P.; Franzen, S. Langmuir 2007, 23 (11), 6053-6062.

(64) Kozak, M. J. Appl. Crystallogr. 2005, 38, 555-558.

(65) Cummins, H. Z. Photon Correlation Spectroscopy and Velocimetry; Plenum Press: New York, 1977.

(66) Schurr, J. M. CRC Crit. Rev. Biochem. 1977, 4 (4), 371-431.

(67) http://www.robinwood.com/Catalog/Technical/Gen3DTuts/ Gen3DPages/RefractionIndexList.html, 2010.

(68) Drexler, K. E. Proc. Natl. Acad. Sci. U.S.A. 1981, 78 (9), 5275-5278.

(69) Kun, R.; Szekeres, M.; Dekany, I. J. Therm. Anal. Calorim. 2009, 96 (3), 1009-1017.

(70) Li, Y.; Lee, J.; Lal, J.; An, L.; Huang, Q. J. Phys. Chem. B 2008, 112 (12), 3797-3806

(71) Lenk, T. J.; Horbett, T. A.; Ratner, B. D.; Chittur, K. K. Langmuir 1991, 7 (8), 1755-1764.

(72) Roach, P.; Farrar, D.; Perry, C. C. J. Am. Chem. Soc. 2005, 127 (22), 8168-8173.

(73) Smith, J. R.; Cicerone, M. T.; Meuse, C. W. Langmuir 2009, 25 (8), 4571-4578

(74) Mulholland, G. W.; Donnelly, M. K.; Hagwood, C. R.; Kukuck, S. R.; Hackley, V. A.; Pui, D. Y. H. J. Res. Natl. Inst. Stand. Technol. 2006, 111 (4), 257-312.

(75) National Institute of Standards and Technology (NIST) Report of Investigation, Reference Materials 8011-8013.

(76) http://www.its.bldrdoc.gov/fs-1037/Federal Standard 1037C, 2007.

(77) Vigil, R. D.; Ziff, R. M. J. Chem. Phys. 1989, 91 (4), 2599-2602.

(78) Adamczyk, Z.; Barbasz, J.; Ciesla, M. Langmuir 2010, 26 (14), 11934-11945.

(79) Cederquist, K. B.; Keating, C. D. ACS Nano 2009, 3 (2), 256-260.

(80) Hill, H. D.; Millstone, J. E.; Banholzer, M. J.; Mirkin, C. A. ACS Nano 2009, 3 (2), 418-424.

(81) Kaiden, K.; Matsui, T.; Tanaka, S. Appl. Spectrosc. 1987, 41 (2), 180-184.

(82) Petkova, G. A.; Kral, V. Bioorg. Med. Chem. 2010, 18 (18), 6651-6656.

(83) Wang, W. R.; Zhu, R. R.; Xiao, R.; Liu, H.; Wang, S. L. Biol. Trace Elem. Res. 2010, DOI 10.1007/s12011-010-8823-x. 